\title{
Epigenome-wide association studies: current knowledge, strategies and recommendations
}

\author{
Maria Pia Campagna ${ }^{1}$, Alexandre Xavier ${ }^{2,3}$, Jeannette Lechner-Scott ${ }^{3,4}$, Vicky Maltby ${ }^{2,3}$, Rodney J. Scott ${ }^{2,3,5}$, \\ Helmut Butzkueven ${ }^{1,6}$, Vilija G. Jokubaitis ${ }^{1,6}$ and Rodney A. Lea ${ }^{3,7^{*}}$ (1)
}

\begin{abstract}
The aetiology and pathophysiology of complex diseases are driven by the interaction between genetic and environmental factors. The variability in risk and outcomes in these diseases are incompletely explained by genetics or environmental risk factors individually. Therefore, researchers are now exploring the epigenome, a biological interface at which genetics and the environment can interact. There is a growing body of evidence supporting the role of epigenetic mechanisms in complex disease pathophysiology. Epigenome-wide association studies (EWASes) investigate the association between a phenotype and epigenetic variants, most commonly DNA methylation. The decreasing cost of measuring epigenome-wide methylation and the increasing accessibility of bioinformatic pipelines have contributed to the rise in EWASes published in recent years. Here, we review the current literature on these EWASes and provide further recommendations and strategies for successfully conducting them. We have constrained our review to studies using methylation data as this is the most studied epigenetic mechanism; microarray-based data as whole-genome bisulphite sequencing remains prohibitively expensive for most laboratories; and blood-based studies due to the non-invasiveness of peripheral blood collection and availability of archived DNA, as well as the accessibility of publicly available blood-cell-based methylation data. Further, we address multiple novel areas of EWAS analysis that have not been covered in previous reviews: (1) longitudinal study designs, (2) the chip analysis methylation pipeline (ChAMP), (3) differentially methylated region (DMR) identification paradigms, (4) methylation quantitative trait loci (methQTL) analysis, (5) methylation age analysis and (6) identifying cell-specific differential methylation from mixed cell data using statistical deconvolution.
\end{abstract}

Keywords: Epigenetics, Methylation, EWAS, ChAMP, Complex diseases, Bioinformatics

\section{Introduction}

Epigenetic mechanisms involve modifications to genomic DNA (both heritable and/or modifiable) that can affect cellular phenotypes and in turn, influence complex disease aetiology and outcomes. The most widely studied epigenetic mechanism is DNA methylation, which can regulate gene expression through the presence or absence of a methyl group on cytosine-phosphate-guanine $(\mathrm{CpG})$

\footnotetext{
*Correspondence: rodney.lea@qut.edu.au

${ }^{7}$ Centre for Genomics and Personalised Health, School of Biomedical Sciences, Queensland University of Technology, Brisbane, Australia Full list of author information is available at the end of the article
}

dinucleotides. Over the last decade, the ability to study methylation at the genome-wide level has led to the application of the epigenome-wide association study (EWASes), which has increased our understanding of the role of methylation in many diseases [1-4]. As genomewide methylation scanning technology has evolved, so too have bioinformatic tools to process, analyse and interpret methylation data from EWASes.

The aim of this review is to perform an up-to-date critical assessment of the tools and strategies available for conducting EWASes, specifically focusing on bloodcell derived methylation data. We focus on blood cells because (1) peripheral blood is one of the least-invasive original author(s) and the source, provide a link to the Creative Commons licence, and indicate if changes were made. The images or other third party material in this article are included in the article's Creative Commons licence, unless indicated otherwise in a credit line to the material. If material is not included in the article's Creative Commons licence and your intended use is not permitted by statutory regulation or exceeds the permitted use, you will need to obtain permission directly from the copyright holder. To view a copy of this licence, visit http://creativecommons.org/licenses/by/4.0/. The Creative Commons Public Domain Dedication waiver (http://creativeco mmons.org/publicdomain/zero/1.0/) applies to the data made available in this article, unless otherwise stated in a credit line to the data. 
tissues to collect, (2) there are many large-scale DNA banks of convenience available for conducting EWASes, (3) the most common publicly available methylation data is from whole blood or whole blood cell subsets, and (4) blood cell pathology is involved in many complex diseases. Furthermore, we review study design and methodological features of EWASes that have not been addressed in the literature previously: (1) longitudinal study designs, (2) the chip analysis methylation pipeline (ChAMP), (3) differentially methylated region (DMR) identification paradigms, (4) methylation quantitative trait loci (methQTL) analysis, (5) methylation age analysis and (6) identifying cell-specific differential methylation from mixed cell data using statistical deconvolution. The goal of this review is to provide researchers with a reference workflow and guidelines for conducting bloodcell-based EWASes.

\section{Epigenome-wide association studies}

The aim of an epigenome-wide association study (EWAS) is to examine genome-wide epigenetic variants (predominantly DNA methylation at CpGs), to detect differences that are statistical associated with phenotypes of interest.

The most common way to study DNA methylation is with bisulphite converted genomic DNA and microarrays. Bisulphite conversion deaminates unmethylated cytosines, producing uracil, on denatured genomic DNA.
Methylated cytosines remain unaffected, and therefore, bisulphite converted genomic DNA contains methylated cytosines only. Microarrays are a collection of different oligonucleotides fixed on a solid substrate (usually glass) that can hybridise to complementary DNA strands. The methylation level is measured at each $\mathrm{CpG}$ present on the microarray and compared between (or within) groups of interest to detect differentially methylated positions (DMPs) and regions (DMRs). A DMP is a single $\mathrm{CpG}$ dinucleotide that is differentially methylated between groups, as determined by statistical significance and effect size thresholds. The definition of a DMR differs between studies based on the algorithm used but can broadly be defined as a region containing multiple DMPs.

The first commercial high-density microarray measuring genome-wide methylation was the HumanMethylation27 (27K) released by Illumina in 2009 [5]. The $27 \mathrm{~K}$ microarray allowed researchers to measure methylation across more than 27,000 CpG sites spanning over 14,000 genes and paved the way for mainstream EWASes. The HumanMethylation450 (450K) microarray followed in 2011 and rapidly gained popularity as it measures methylation at over $450,000 \mathrm{CpG}$ sites [6]. The $450 \mathrm{~K}$ remains the most cited Illumina microarray for DNA methylation studies to date (Fig. 1). In 2016, Illumina produced a new iteration of the $450 \mathrm{~K}$, called the HumanMethylation 850 (EPIC) microarray, which measures methylation at over

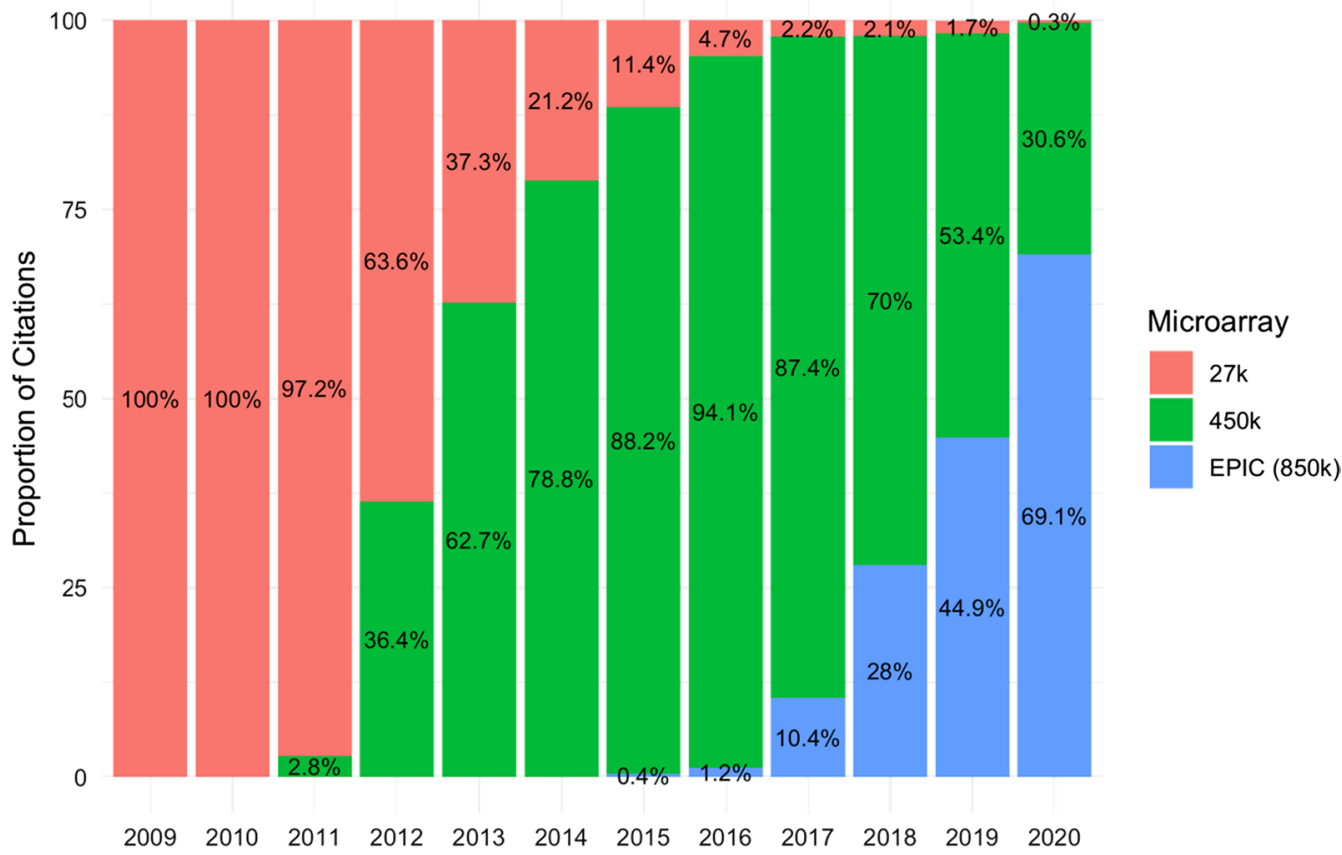

Fig. 1 Popularity of methylation microarrays. The proportion of EWASes deposited on GEO (NCBI) each year, by array type. Abbreviations: EWAS = Epigenome-wide association study, GEO= Gene Expression Omnibus, NCBI = National Center for Biotechnology Information 
850,000 CpG sites [7]. Illumina microarrays predominantly measure methylation in gene promoter regions. The coverage of intergenic regulatory regions has improved with each iteration of the microarray; however, the EPIC microarray still only covers $58 \%$ of FANTOM enhancers, $27 \%$ of proximal regulatory elements and $7 \%$ of distal regulatory elements [7]. Notably, Agilent now produces microarrays that measure methylation at over 235,000 CpG sites; however, these microarrays are not cited as frequently as Illumina microarrays. Nimblegen also produced microarrays, but these were discontinued in 2012.

The ability to measure methylation in a high-throughput manner drove the development of bioinformatic pipelines. These have streamlined analyses and overcome the hurdles that arise from highly dimensional datasets. Two main bioinformatics analysis packages for EWAS data are Minfi [8] and ChAMP [9], which emerged as open-source alternatives to GenomeStudio, the original proprietary tool provided by Illumina. Both packages were released in 2014 and were built to analyse 450k microarray data. In 2017, they were updated to also include EPIC microarray data $[10,11]$. Minfi is the most cited tool for $450 \mathrm{k}$ data analysis, while ChAMP is becoming the most cited tool for EPIC data analysis (Fig. 2). Minfi and ChAMP allow users to import data files directly produced from methylation microarrays (i.e. raw. idat files), perform quality control (QC), normalisation and detection of both DMPs and DMRs. Different downstream analyses are available for each package (Fig. 3).

\section{Study designs}

EWASes can be conducted using unrelated case-control and longitudinal designs, as well as one sample quantitative trait and family-based study designs. Here, we compare and contrast case-control and longitudinal study designs and discuss other important considerations such as power and sample size. Quantitative trait and familybased study designs differ substantially from those of non-related individuals and have been reviewed in detail previously [12]. The main difference between case-control and longitudinal study designs is the practicality and affordability of case-control studies in comparison with longitudinal studies. However, only longitudinal studies can assess the relationship between changes in epigenetics and phenotype during the course of disease. Notably, case-control studies investigate the relationship between dichotomous traits and methylation using contrast comparisons.

\section{Case-control designs}

Case-control EWASes are the most employed study design. The case-control design is a standard design in epidemiology and involves grouping unrelated

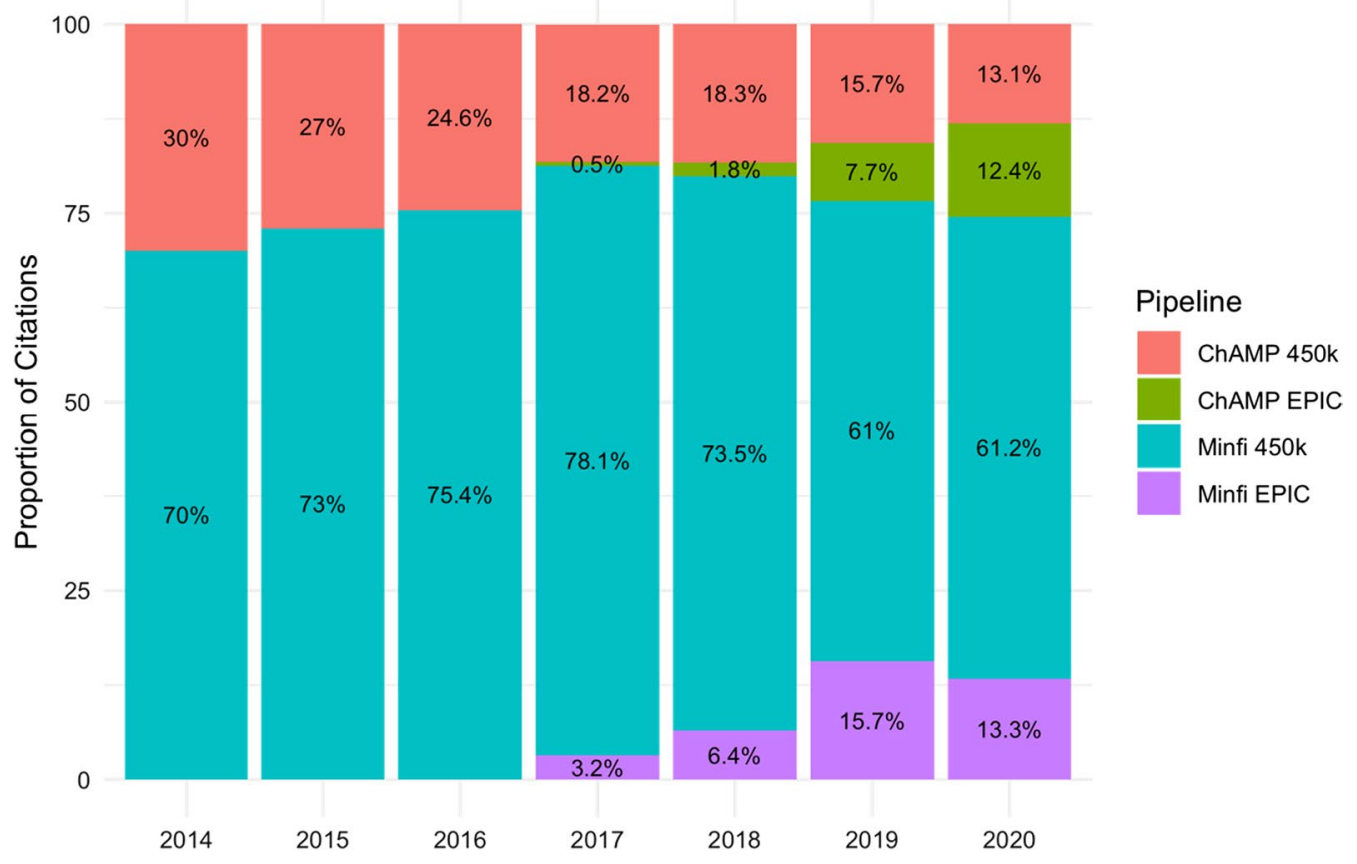

Fig. 2 Popularity of EWAS pipelines. The proportion of PubMed citations by year for methylation data analysis using different Illumina arrays. Abbreviations: EWAS = Epigenome-wide association study 

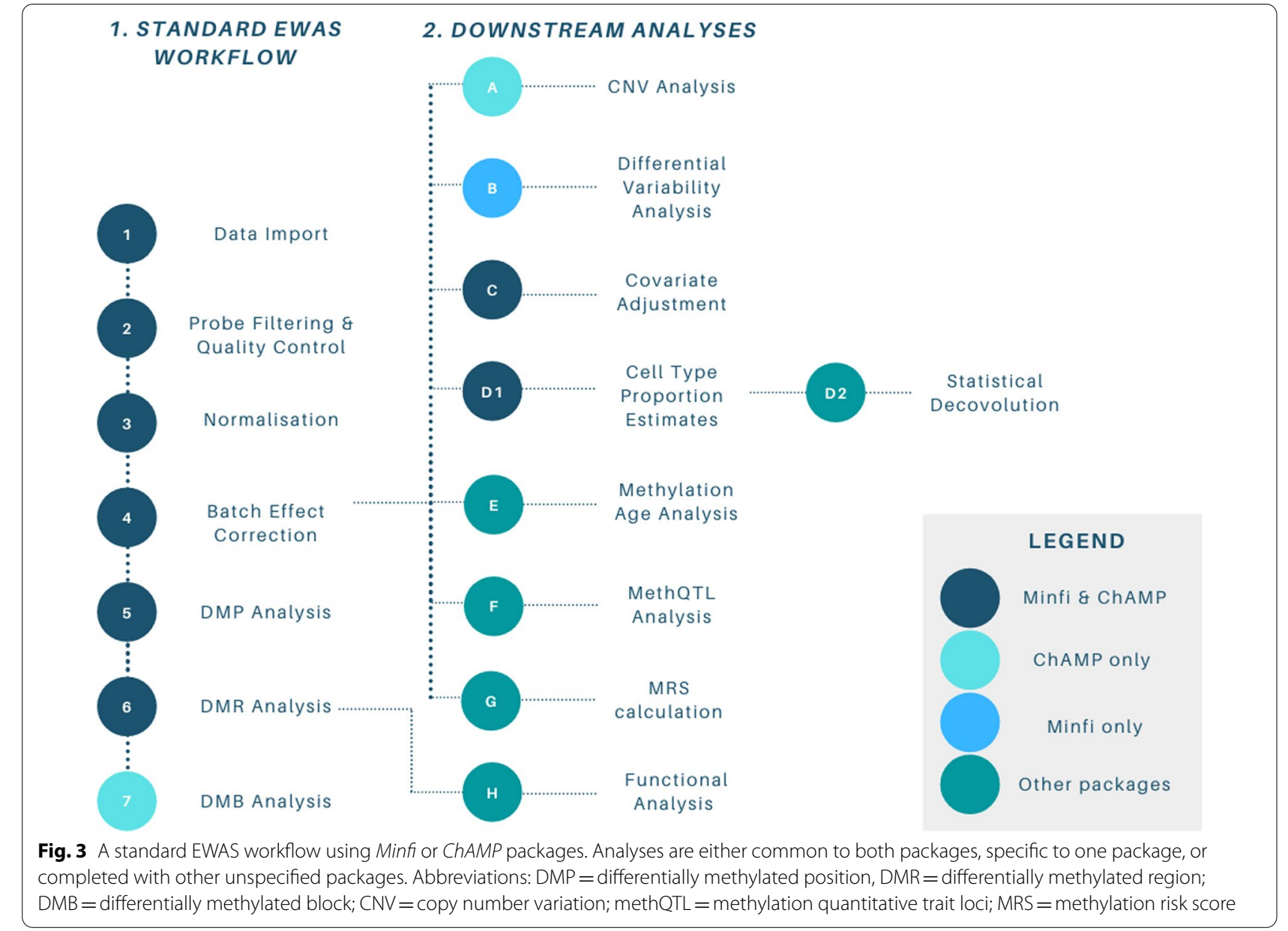

participants into a phenotype of interest, such as the presence of disease, and compares CpG methylation levels to a group of subjects without the phenotype (i.e. a control group) [13-16]. The control group can be matched for potential confounding factors such as age, sex, ethnicity or genotype at a locus that has previously been associated with the phenotype of interest. Subjects are usually collected in a cross-sectional manner from the population of interest making this design the most feasible in terms of logistics and cost (discussed below in "Longitudinal designs" section). For blood-based EWAS, researchers can utilise existing DNA biobanks from past case-control genome-wide association studies (GWASes) [17]. Thus, the main benefit of this study design is the ability to obtain large subject numbers.

The primary limitation of the case-control study design is the inability to determine the timing of the relationship between differential methylation and phenotype. That is, whether differential methylation between cases and controls results in, or is a result of, disease. For this reason, case-control EWASs are typically restricted to claims to association rather assigning cause or effect of the relationships. Auxiliary approaches such Mendelian randomisation (MR) can be used within the case-control designs to statistically infer cause and effect between CpGs associated with the phenotype. MR uses genetic variants that are associated with the same CpGs and/ or the phenotype to reveal potential causal associations [18]. However, prospective longitudinal study designs are required to truly understand the timing and mechanism behind phenotype-inducing changes in methylation.

\section{Longitudinal designs}

Longitudinal studies allow researchers to determine intra-individual trajectories (changes) as well as interindividual variability in methylation and/or phenotype over time. However, they are more difficult to set up than case-control studies. These studies follow groups of individuals over months, years or decades measuring methylation, and if possible, phenotype, at multiple timepoints. The commonest form of longitudinal studies in the EWAS literature are natural history studies, which track methylation trajectories from birth in healthy individuals [19-24]. However, it is harder to establish longitudinal 
studies following disease states, as pre-disease onset samples are very difficult to collect. A significant advantage of the longitudinal study design is the ability to track methylome changes in relation to time and phenotype, possibly allowing causal relationships to be established. Nevertheless, the time and cost associated with longitudinal studies remain prohibitive for many researchers. Consequently, the majority of longitudinal EWASes study healthy, natural history cohorts.

Longitudinal studies in natural history cohorts demonstrate the dynamic nature of DNA methylation throughout the lifespan, particularly in the early years of life. During the first five years of life, the methylome undergoes drastic remodelling with a tendency towards global hypermethylation [19-24]. Methylation changes predominantly occur on autosomal chromosomes [19-23], with hypermethylation in CpG dense regions, including gene promoters, intragenic regions and transcription start sites [20-23]. Hypermethylated genes are overrepresented in developmental functions such as tissue morphogenesis, haematological system development, the effector immune response, neuronal-related functions and cell-cell signalling [21-23]. Hypomethylation occurs in CpG sparse regions [20,22, 23], primarily in immune response-related genes including antigen binding and intracellular signalling, cellular components related to the major histocompatibility complex (MHC) protein complex and cytoskeleton, as well as messenger RNA and protein metabolism [21-23]. Notably, leukocytes undergo major epigenome remodelling in the first five years of life, potentially indicating an "immunological window of opportunity" in childhood [22, 25]. In individuals over 50 years of age, inter-individual variation and intra-individual demethylation tend to increase with time [19, 26-28]. Age-related demethylation is particularly pronounced at Alu repetitive elements (conserved regulatory regions), which correlate with genome-wide methylation levels [27].

Longitudinal study designs have also been used to study epigenetic drift in monozygotic (MZ) and dizygotic (DZ) twin pairs. In twins, epigenetic drift refers to the diverging epigenomes over time, as they are exposed to different environmental factors [15-18], and is more pronounced in DZ than MZ twins [28-31]. As MZ twins are genetically identical, they are useful for studying the impact of environmental factors on the epigenome over time. Such studies have shown that genetics explains less than $24 \%$ of variation in epigenetic drift, demonstrating a substantial impact of environmental factors on the epigenome [29]. Epigenetic drift also attenuates with age, and primarily affects genes enriched for immune and inflammation pathways [29]. In summary, genetics contributes to the methylome's stability over time, while environmental factors contribute to epigenetic drift.

In the current EWAS literature, longitudinal studies typically examine changes in methylation across two time points only. As such, methodological strategies for conducting studies with multiple time points are lacking. Furthermore, these studies have used data derived from the 450k microarray, which is enriched for CpGdense genomic regions ( $\mathrm{CpG}$ islands). There is growing evidence that methylation in CpG-sparse regions, such as enhancers and gene bodies, has significant functional consequences through altering gene expression [32]. Hence, longitudinal studies with multiple time points using the EPIC microarray could provide novel insights into methylation trajectories in health and disease.

\section{Replication or validation analyses}

Replication or validation analyses are critical for EWASes to confirm preliminary findings. This is particularly pertinent in epigenetic studies as a range of confounding environmental exposures-known and unknown-may be present. As with GWASes, independently ascertained replication cohorts are required to confirm (or refute) preliminary results and establish effect sizes, and therefore inform pathology or clinical utility (e.g. biomarkers). Replication is defined as the reproducibility of preliminary results in a cohort that is as similar to, but independent of, the preliminary cohort. For this reason, it is often difficult-and sometimes impossible-for researchers to obtain a suitable replication cohort. This is especially true for rare diseases where patient numbers are difficult to build up. In this instance, there are several approaches researchers can take to validate preliminary results. Validation is defined as corroboration of results in a cohort, or using a dataset, that does not originate from the discovery phase of the study. In EWASes, validation can be achieved by (1) corroborating preliminary findings in a similar, although not identical cohort, or general population, (2) confirming that preliminary findings are not corroborated in a healthy or natural history cohort or dataset, indicating disease specificity, (3) utilising EWAS databases (see "Epigenome-wide association study databases" section) to access raw.idat files and/or summary statistics for validation analyses, (4) using the literature to provide biological or pathological support for preliminary results or (5) using animal studies to gain specific mechanistic insight. For animal studies, rodents are useful as they age faster than humans and researchers can control for environmental exposures and confounders.

\section{Power and sample size considerations}

EWASes of complex diseases must be statistically powered to identify modest, but important, differences in 
DNA methylation between groups or time points (i.e. effect sizes). Statistical power is defined as the probability that a statistical test rejects the null hypothesis when the alternative hypothesis is correct. Therefore, in adequately powered studies, there is a reduced risk of type I (false positive) and type II (false negative) errors. Significance thresholds, sample size and effect size all affect statistical power of EWASes.

In EWASes, Type I error rates (significance thresholds) are impacted by multiple testing as a vast number of CpG sites are analysed. Bonferroni corrected significance thresholds are sometimes used to overcome the multiple testing burden. However, Bonferroni deflation for the number of CpG sites tested is widely considered to be too stringent in EWASes as methylation is highly correlated across the genome. Thus, the actual number of independent tests is far fewer than the number of CpGs on each microarray [33]. Therefore, a false-discovery rate (FDR) threshold is commonly used, which provides a balanced compromise between type I and type II error rates (i.e. FDR $<0.05$ ). However, the heteroskedasticity of methylation data (i.e. non-constant variance of methylation levels between groups) and non-uniform distribution of p-values across measured CpG sites can violate FDR assumptions. Thus, using an FDR threshold could limit the reproducibility of results across studies [33]. To overcome the limitations of both Bonferroni corrected $\mathrm{p}$-values and FDR thresholds, simulation studies have calculated the number of independent tests in EWASes using methylation data from the $450 \mathrm{k}$ and EPIC microarrays. An unadjusted significance threshold of $9.42 \times 10^{-8}$ $\left(95 \% \mathrm{CI}=2.97 \times 10^{-8}-1.49 \times 10^{-15}\right)$ is recommended for analyses using EPIC data [33], and $2.4 \times 10^{-7}$ (95\% CI not reported) for analyses using $450 \mathrm{k}$ data $[34,35]$. The ChAMP pipeline (outlined below in "The chip analysis methylation pipeline (ChAMP)" section) calculates unadjusted and adjusted p-values (FDR), allowing researchers to use the significance threshold that is most suitable for their study.

As complex phenotypes rarely have effect sizes larger than $5 \%$, the most straightforward way to increase power is to increase sample size. EWAS power studies using case-control [33-35] or family-based [34, 35] simulations recommend a sample size of 1000 (500 cases and 500 controls) to detect statistically significant DMPs and DMRs. However, in some cases these are not required. For example, due to large methylation differences in the Human Leukocyte Antigen (HLA) region, several EWASes of multiple sclerosis have identified differential methylation of $>20 \%$ between cases and controls with sample sizes of 20-30 per group [13-15]. There are currently no published sample size recommendations for longitudinal EWASes. However, longitudinal studies in
Parkinson's disease and Type I diabetes have identified differential methylation between groups with sample sizes ranging from 85 to 190 per group [36, 37]. Large sample sizes may be difficult to obtain in longitudinal studies, due to costs and attrition rates.

\section{The chip analysis methylation pipeline (ChAMP)}

ChAMP is a Bioconductor package that provides a powerful tool for analysis of DNA methylation data obtained with the Illumina $450 \mathrm{~K}$ or EPIC microarrays [11]. It is designed for the R statistical environment [11], and integrates existing pre-processing and analysis tools, such as Minfi [8], into a single pipeline. The ChAMP pipeline consists of eight main functions, which can be executed in full with the command champ.process(). Nevertheless, we recommend completing each function separately so that researchers can assess the interim results and tailor the parameters of each function to best suit their analysis [11]. The steps and recommended tools for primary EWAS analyses discussed below are summarised in Fig. 4.

ChAMP is designed for case-control designs, i.e. identifies differential methylation between two categorical groups. This is also adequate for case-control studies and longitudinal studies with two time points. However, ChAMP is not a suitable package for studies of multiple groups including longitudinal studies with more than two time points. For such analyses, researchers will need to use the individual packages incorporated into ChAMP, such as limma for DMP analysis and DMRcate for DMR analysis. ChAMP is also unsuitable for studies of continuous phenotypes. There are currently no purpose-built tools for this type of analysis; therefore, researchers will need to use alternate statistical approaches, such as linear regression modelling between $\mathrm{CpG}$ and phenotype within a suitable statistics program (e.g. $\mathrm{R}$ environment [38]).

\section{Data pre-processing Probe filtering}

ChAMP offers two functions to load data from.idat files and a corresponding sample sheet: champ.import() and champ.load(). The former uses Minfi functions to return objects (e.g. data matrices) fed directly into downstream normalisation functions [8], while the latter imports and filters data in one step [11]. Alternatively, researchers can use champ.filter() to customise the following parameters: (1) detection p-values $(p>0.01)$, (2) low-quality probes ( $<3$ beads in $\geq 5 \%$ of samples per probe), (3) probe type (non-CpG probes), (4) chromosomal location (non-autosomal), (5) presence of single-nucleotide polymorphisms (SNPs) in the probe sequence (populationspecific) [5], (6) cross-hybridisation [39] and (7) multi-hit 


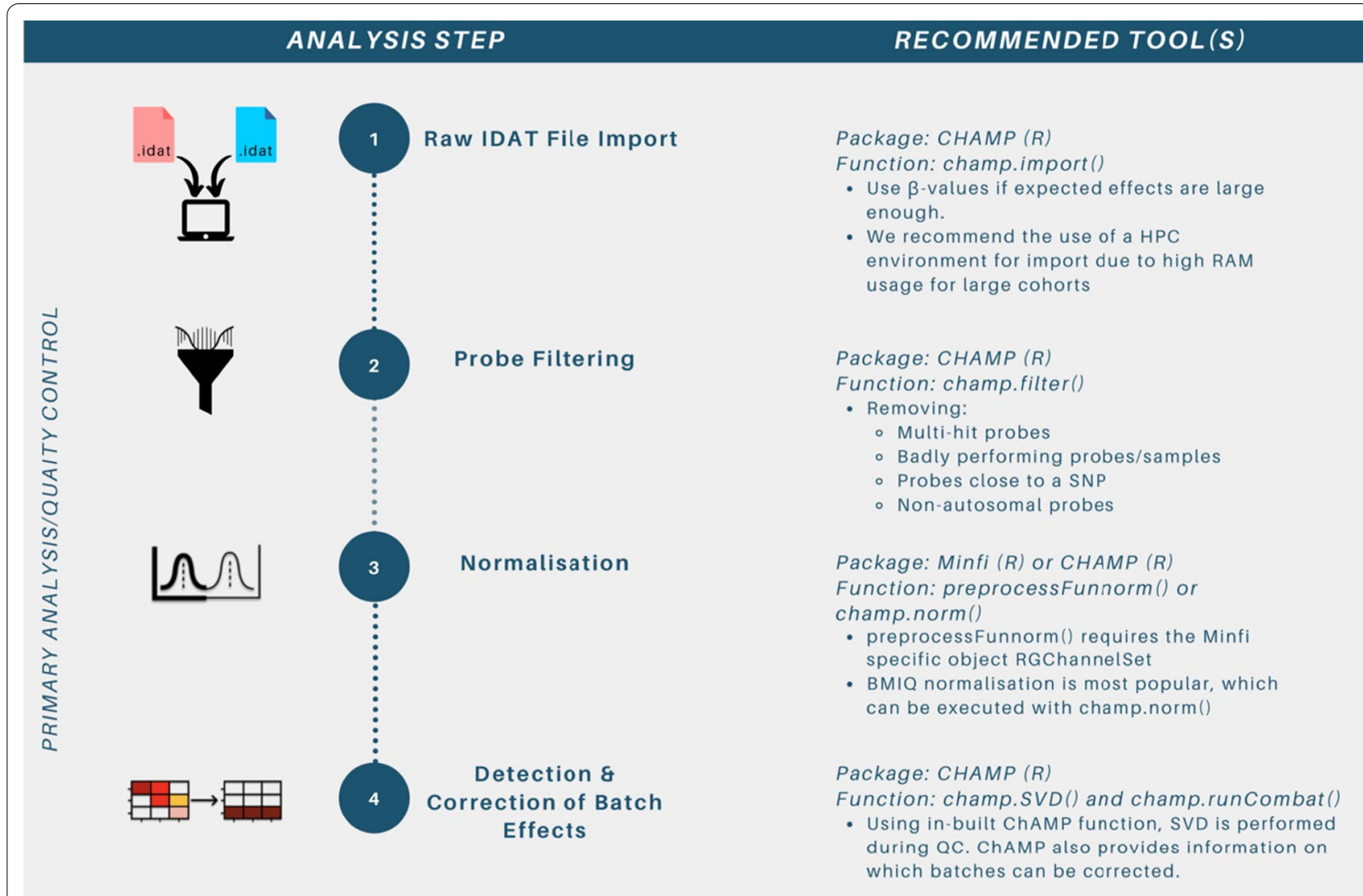

\title{
ANALYSIS READY BETA OR M-VALUE MATRIX
}

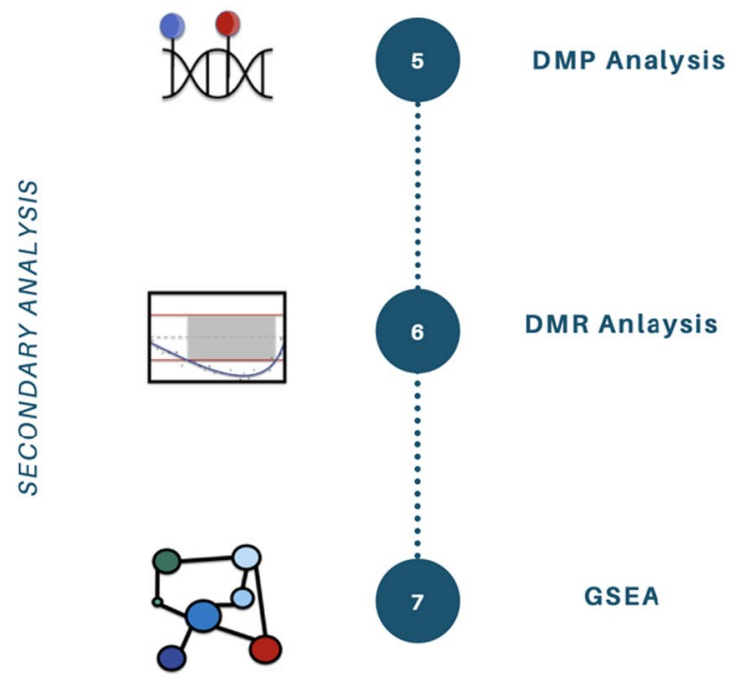

\author{
Package: CHAMP (R) \\ Function: champ.DMP() \\ - 1st Approach: Use the ChAMP.DMP built in \\ function \\ - 2nd Approach: Use glm (with binary \\ phenotype as outcome) or Im (with CpG \\ methylation as outcome) including \\ covariates. \\ - Prioritise DMPs with at least 5\% ADB.
}

\section{Package: $C H A M P(R)$}

Function: champ.DMR()

- Use champ.DMR() with the DMRcate method

- Sensible defaults are:

- At least 3 probes per DMR

- $500-2000$ bp distance between probes

- Prioritise DMRs with at least $5 \%$ average ADB

\section{Package: missMethyl (R) \\ Function: goMeth() and goRegion() \\ - For goMeth: Use list of significant probes with at least $1 \cdot 2 \%$ ADB \\ - For goRegion: Use list of significant DMRs with at least $1-2 \%$ average $A D B$}

Fig. 4 Steps and tools for primary EWAS analysis steps. Listed tools include ChAMP (https://bioconductor.org/packages/release/bioc/html/ChAMP. html), Minfi (https://bioconductor.org/packages/release/bioc/html/minfi.html) and missMethyl (http://bioconductor.org/packages/release/bioc/ html/missMethyl.html). Abbreviations: EWAS = epigenome-wide association study, ChAMP = chip analysis methylation pipeline, HPC = high performance computer, RAM = random access memory, SNP = single nucleotide polymorphism, BMIQ = beta mixture quantile, SVD = singular value decomposition, $\mathrm{QC}=$ quality control, $\mathrm{DMR}=$ differentially methylated region, $\mathrm{ADB}=$ absolute deta beta 
probes (probes with unspecific genomic alignment) [40]. Default filtering thresholds are adequate for most analyses. The dataset used for cross-hybridised probe filtering with ChAMP is from an early study which interrogated the $450 \mathrm{k}$ microarray [40]. More recently, 15,000 additional cross-hybridised probes were identified for the EPIC microarray [41]. Therefore, it is imperative that researchers using the EPIC microarray further filter data for the additional cross-hybridised probes prior to data normalisation.

\section{Beta- and $M$ values}

ChAMP accommodates the use of either beta- or $\mathrm{M}$ values representing $\mathrm{CpG}$ methylation. Beta-values are an index of methylation levels and range from zero (completely unmethylated) to one (completely methylated). Notably, methylation beta-values differ to beta coefficients produced by regression models. Beta-values are the ratio of the methylated probe intensity and overall probe intensity (the sum of methylated and unmethylated probe intensities). Methylation beta-values are easy to interpret as they can be converted to a percentage of methylation ranging from 0 (unmethylated) to $100 \%$ (methylated). One drawback of beta-values is that they follow mixed statistical probability distributions (i.e. beta-binomial), which can cause issues for conventional linear regression models whereby CpG beta-values are modelled as the dependent variable. For this reason, beta-values may be transformed into $M$ values, which are the $\log ^{2}$ ratio of the methylated probe intensity and the unmethylated probe intensity. Negative $M$ values indicate lower methylation, whereas positive $M$ values indicate higher methylation. Although $M$ values are considered statistically more appropriate than beta-values when the CpG is modelled as the dependent variable than betavalues, they lack intuitive interpretation [42]. Detailed assessment of the use of beta- or $\mathrm{M}$ values has been published elsewhere [42].

\section{Probe imputation}

Imputation is the process of inferring beta-values for missing probes in a beta matrix using statistics and machine learning. Missing probes can result from inadequate assays or across microarray iterations. For example, probes from the $450 \mathrm{~K}$ microarray compared to the EPIC microarray can be imputed for direct comparison. Imputation is particularly useful in longitudinal studies to harmonise data collected with different platforms across timepoints due to technological development.

champ.impute() imputes missing beta-values in a filtered beta matrix (i.e. after champ.load()) via three methods: removal of the missing probes, k-nearest neighbours or a combination of both [11]. K-nearest neighbour is a machine learning method in which a Euclidean metric is used to identify neighbouring probes (to the missing probe), and the beta-value of the missing probe is imputed as the average of the beta-values at the neighbouring probes [11]. We recommend the removal of missing probes for small sample sizes and a combination of removal and k-nearest neighbours for larger sample sizes.

\section{Data normalisation}

Illumina microarrays use two distinct hybridisation chemistries to measure methylation at probes, Type I and Type II, which produce different beta-value distributions. These distributions must be normalised to avoid the biased detection of DMPs enriched for Type I probes. Beta-mixture quantile normalisation (BMIQ) [43] is the default normalisation method in ChAMP, but other options are available, including subset-quantile within microarray normalisation (SWAN) [44], peak-based correction (PBC) [45] or functional normalisation (Funnorm) [46]. All of these methods have been previously described in detail [47-51]. For analyses where there is a strong association between methylation and phenotype, each method performs similarly in terms of accuracy [50, 51]. However, Funnorm produces the most replicable results in analyses where global methylation changes are expected; for example, in case-control, between-disease studies or inter-tissue comparisons. The same method also removes a large amount of technical variation in the unsupervised normalisation process, improving downstream batch effect correction. Nevertheless, BMIQ remains the most popular normalisation method to date (Fig. 5).

It is not uncommon for researchers to use methylation data derived from different microarrays. For example, in case-control studies, cases may be prospectively recruited and analysed using the EPIC microarray, while historical $450 \mathrm{k}$ microarray data may be used for controls. In longitudinal analyses, the $450 \mathrm{k}$ microarray may be used at baseline, while the EPIC microarray is used at follow-up timepoints. In this scenario, the single-sample noob (SSnoob) function in Minfi is the most useful tool for normalisation [10]. SSnoob integrates $450 \mathrm{~K}$ and EPIC microarray data for joint normalisation, outperforming BMIQ and Funnorm in this process [10].

\section{Addressing technical variation (batch effects)}

The default method for batch effect identification in ChAMP is singular value decomposition (SVD) [9]. Basically, SVD correlates principal components with biological and technical variation using information from the user-provided sample sheet [25]. 


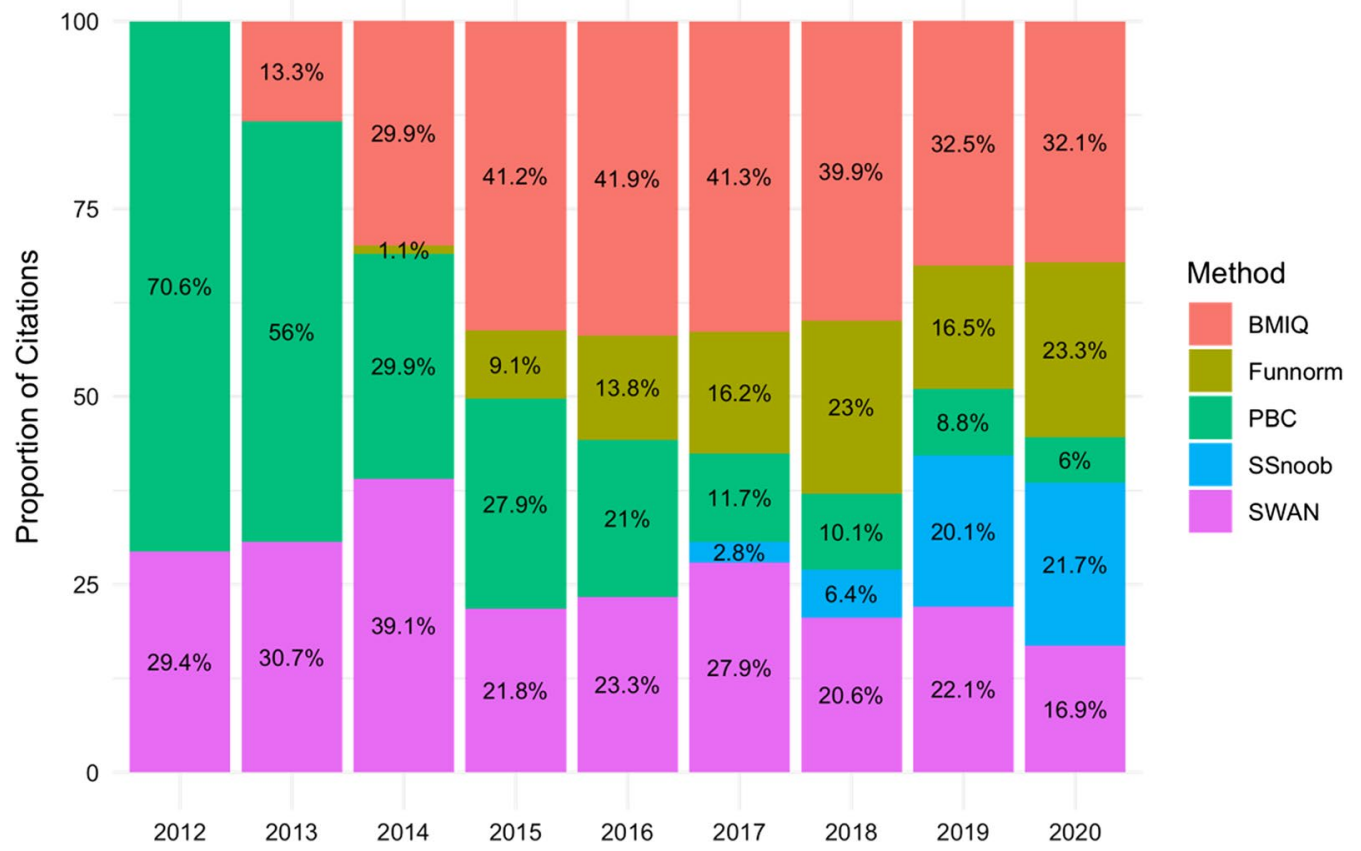

Fig. 5 Popularity of normalisation methods. The proportion of PubMed citations by year for methylation data normalisation algorithms.

The default method for batch effect correction in ChAMP is ComBat [9], which uses an empirical Bayes method to correct batch effects. ComBat requires two inputs: (1) the variables which should not be adjusted (i.e., biological variation caused by the variable of interest), and (2) the batch variables to be adjusted for (for example, slide, microarray, plate), previously identified in SVD analysis. Researchers should be aware that ComBat can introduce false signals if biological and technical variation are partially or wholly confounded [52-55]. In a case-control study, this may occur if all samples from cases run on the same plate and controls on another, thus causing systematic technical error via batch effects. The analogous error in a longitudinal study is running samples from each time point on different plates. To reduce the risk of false signals, ChAMP checks that technical and biological variation is not confounded before adjustment [11]. False signals can be minimised a priori by manually randomising samples across microarrays and excluding biological covariates from batch effect correction [55]. In an ideal experiment, all samples would be processed in one batch; however, this is impossible for large sample sizes. The nature of longitudinal study designs means that they are at higher risk of batch effects than case-control studies, as samples are collected and processed at different time points. Thus assays, microarrays and techniques may evolve, leading to technical variation. ComBat has been shown to be effective in correcting technical variation in longitudinal gene expression studies [56]. Biological and clinical covariates likely influence methylation patterns and should be addressed in downstream statistical analyses rather than at the data pre-processing stage [55]. Notably, for researchers correlating methylation data with other clinical datasets, ComBat has utility in correcting batch effects in various datasets, including gene expression and imaging data [57-59]. Alternatively to ComBat, the missMethyl package uses Remove Unwanted Variation (RUV). The RUV uses negative controls on the microarray to identify and remove unwanted variation $[60,61]$. As a rule of thumb, it is reasonable to move onto downstream analyses if the first two principal components identified with SVD explain more than $80 \%$ of the covariance in the data, and if these are adequately corrected for prior to progressing [11].

\section{Statistical association testing}

The statistical association between methylation levels (beta- or $M$ values) and phenotype are identified at the level of individual CpG sites (DMPs) and broader genomic regions (DMRs). Typically, DMP analyses are straightforward association tests between methylation beta-values and phenotype, whereby the specific statistical test used will depend on the study design and cohort characteristics. DMR analysis is more complex and as such, multiple algorithms for DMR detection are available. 


\section{Differentially methylated positions (DMPs)}

DMPs are CpG sites with statistically significant differences in average (usually mean) methylation levels between groups. ChAMP can identify DMPs between two categorical groups of interest: case-control studies, and longitudinal studies with two time points. For longitudinal studies that collect data for more than two time points, there are different ways of approaching analyses, to identify intragroup changes between time points and/ or intergroup differences over time. For the former, methylation data from the same group at two time points can be analysed as if it was comparing two separate groups using general linear models with repeated measures. For longitudinal studies with more than two time points, linear mixed models (LMMs) including time as a random effect term can be used to compare methylation at each timepoint simultaneously. LMMs are useful in longitudinal studies where missing data is common, or to address differences in the timing of measurements between groups. To identify intergroup differences in change over time there are multiple statistical approaches available: (1) use ChAMP to identify DMPs at baseline and re-analyse methylation differences at only these sites at subsequent time points to identify the methylation trajectory (change) at these sites over time, (2) use ChAMP to identify DMPs at each timepoint independently, and correlate results between timepoints, noting which DMPs are variable (significantly different at any time point) or consistent (significantly different at each time point), and their direction of effect or (3) use LMM with a group contrast term to assess the relationship between change in methylation over time and phenotype group.

The champ.DMP() function in ChAMP uses the Bioconductor package limma [62] to identify DMPs from a beta-matrix. This package conducts a pairwise comparison of beta-values between groups, by fitting the same general linear model to each probe separately and computing a moderated t-statistic and unadjusted $p$ value. A moderated t-statistic is the ratio of the beta-value for a $\mathrm{CpG}$ to a pooled standard error (SE). By pooling information from all CpGs limma moderates SE at individual probes to improve inference about each CpG. ChAMP subsequently corrects unadjusted $p$ values for multiple testing using the Benjamini and Hochberg method [63] and reports an FDR. The use of unadjusted and adjusted significance values by ChAMP allows researchers the option of significance thresholds to guide the interpretation. However, strict adherence to significance thresholds may lead researchers to overlook DMPs with significant biological effects (i.e. incur Type II error). Therefore, if few DMPs surpass default significance thresholds, we recommend the use of secondary criteria applied in a stepwise manner: (1) identify DMPs with an unadjusted $p$ value below $0.05,(2)$ identify DMPs with effect sizes larger than $10 \%$, (i.e. an absolute delta beta-value $>0.1$ ). If there are still no, or few, hits researchers may then relax the effect size threshold further in the interests of detecting minor effect DMPs. Studies show $<1 \%$ variation in beta-values across technical replicates using the EPIC microarray [6]. This suggests that at least some CpGs with effect sizes below this will be affected by technical variation, rather than biological variation. As such, we recommend a conservative approach of removing DMPs with effect sizes below $2 \%$. However, reducing the threshold to $1 \%$ may still be adopted if desired and yield some important, albeit modest, biological insights. Numerous published studies have used these secondary criteria to identify methylation differences between cases and controls in various diseases and cell types [13-15, 64].

Covariates (e.g. age and sex) can be included in DMP analysis as secondary analyses run outside of ChAMP, using packages like limma or base $\mathrm{R}$ functions. We recommend against including covariates in the primary DMP analyses to detect unadjusted main effects of $\mathrm{CpG}$ on phenotype and using this as a benchmark. However, if sensitivity tests demonstrate an association between covariates and phenotype and/or covariates and methylation, then subsequent multi-factor models can be applied to determine the modification effect of covariates on the DMP. If the DMP signal is modified, we further recommend conducting interaction analyses, but warn that unnecessary inclusion of covariates can overburden the model and lead to reduced statistical power.

\section{Differentially methylated regions (DMRs)}

DMRs are genomic regions made of several contiguous DMPs. They are often associated with a specific gene region, such as $\mathrm{CpG}$ islands in promoter regions, but can also be in intergenic regions. Compared to DMPs, DMRs may be more biologically relevant and are more likely to be associated with modified gene expression because of the strong correlation among adjacent CpGs [65]. Therefore, accurate DMR identification is critical to enable a thorough understanding of the extent of localised differential methylation in relation to the phenotype of interest. There are multiple definitions, approaches and bioinformatic tools available for DMR identification. Thus, we recommend a workflow for identifying statistically and biologically significant DMRs using any of the bioinformatic tools reviewed below.

DMR identification paradigm A preliminary approach identifies consecutive (or closely adjacent) DMPs with the same direction of effect (i.e. all hypomethylated or hypermethylated), that yield some evidence of statistical significance based on $p$ values $[14,15,66]$. For this approach 
we suggest filtering DMPs by an FDR $<0.05$ to identify statistical evidence of association with the phenotype. This threshold is preferred over filtering based on an epigenome-wide threshold of $9.8 \times 10^{-8}$, as it may be overly stringent and increase the risk of Type II error (false negatives). For DMR calling using this approach, researchers should decide the maximum genomic distance between DMPs (i.e. window size) and the minimum number of DMPs required for a DMR to be classified. The overwhelming majority of EWASes use a distance of 1000bp between probe to separate DMRs, regardless of the algorithm [67-70]. Some studies have applied more stringent (500bp) [71] or lenient (2000bp) windows [72]. We recommend a window size of 500-2000bp containing and at least two DMPs for a DMR to be classified, whereby the window is broadened or narrowed accordingly. For example, researchers can broaden the window size in genomic regions of low probe density. These thresholds are guided by the density of $450 \mathrm{~K}$ and EPIC microarrays (see Fig. 6). A window of 500-1000 bp will cover most functional domains of genes, including transcription starting site (TSS) (known to modulate gene expression), $5^{\prime}$ untranslated region $\left(5^{\prime} \mathrm{UTR}\right)$ and first exon. However, a window of 1000 to 2000 bp should allow researchers to identify DMRs located in region scarcely covered: gene body, $3^{\prime}$ untranslated region ( $3^{\prime}$ UTR) and intergenic regions (IGR).

Several tools provide a more programmatic approach for identifying DMRs. Most also depend on defining the DMP threshold, genomic distance between DMPs and minimum number of DMPs per DMR. Bumphunter is one of the most widely used tools, created in 2012 for EWASes using high-density microarrays and large sample sizes [65]. DMRs are detected through "bump hunting" or "peak detection". For each CpG site, Bumphunter produces a slope (or curve) from a linear model based on the phenotype. The smoothed curve is then plotted and analysed for "bumps" that surpass a predefined threshold. Bumphunter's algorithm also incorporates covariates, and variables contributing to technical variation. However, BumpHunter has been shown to lack power and precision [73]. Published in 2015, DMRcate [74] is the most popular tool for DMR detection (as of 2021). It performs a regression of methylation level at each CpG site based on phenotype, accounting for covariates. This is followed by Gaussian smoothing (effect averaging) and grouping nearby CpG sites according to a user-defined window. Despite being more computationally intensive than Bumphunter, DMRcate outperforms Bumphunter and, therefore, has been the tool of choice for DMR identification since 2018 (Fig. 7). While not specific to methylation data, Comb- $p$ [75] is also a popular tool to identify DMRs as its performance is comparable to DMRcate. Comb- $p$ analyses and corrects $\mathrm{p}$ values in a user-defined genomic window based on weighted neighbouring probes, and then assigns a p value to the whole genomic region. Lastly, Probe Lasso [76] is a tool packaged with the ChAMP pipeline. This uses a novel variable window approach to identify larger DMRs in regions with lower probe density-such as intergenic regions. However, it does not allow covariates to be incorporated.

In a recent benchmark study comparing all primary DMR tools (Bumphunter, Comb-p, DMRcate and Probe Lasso), Comb- $p$ was recommended for DMR identification as it has an adequate balance between precision

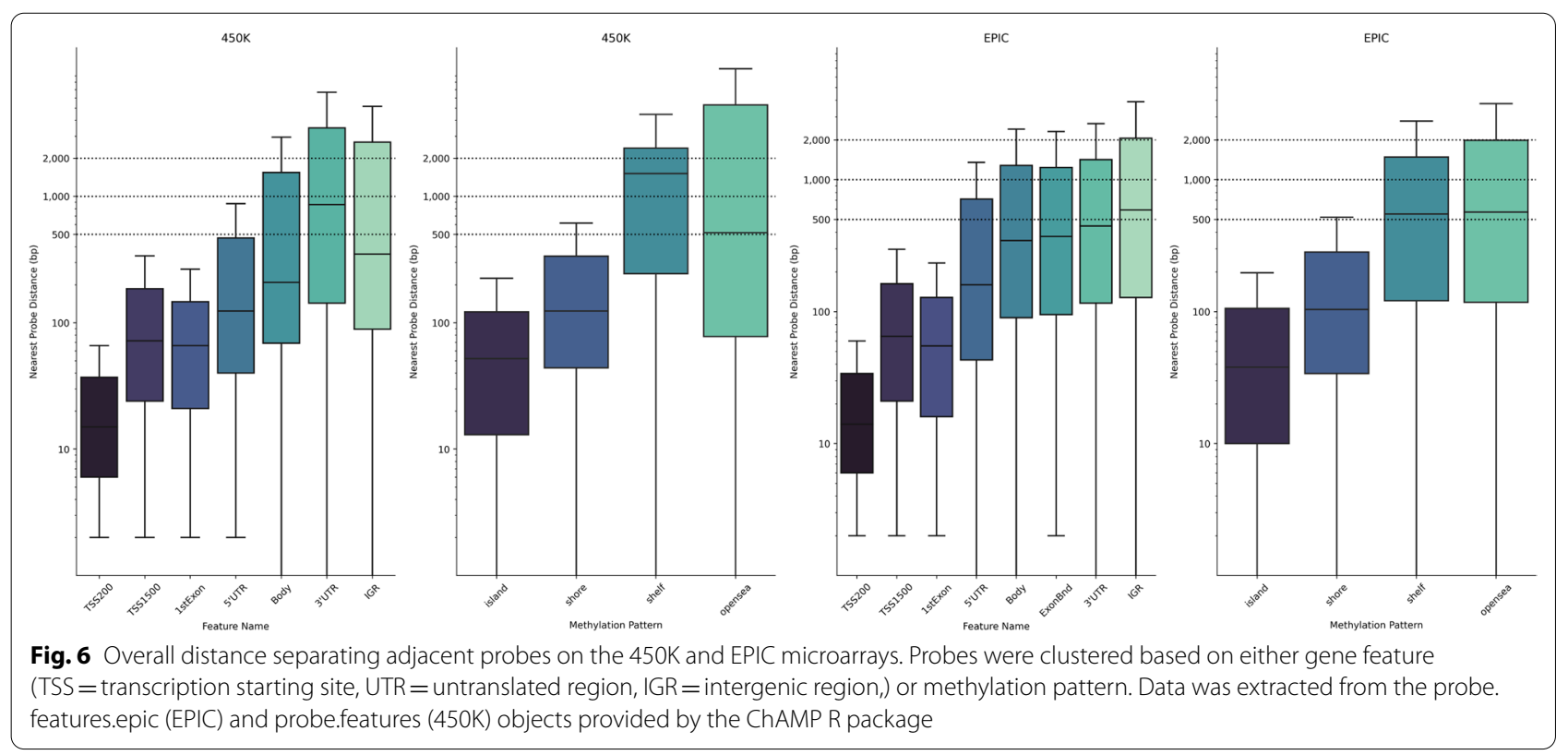




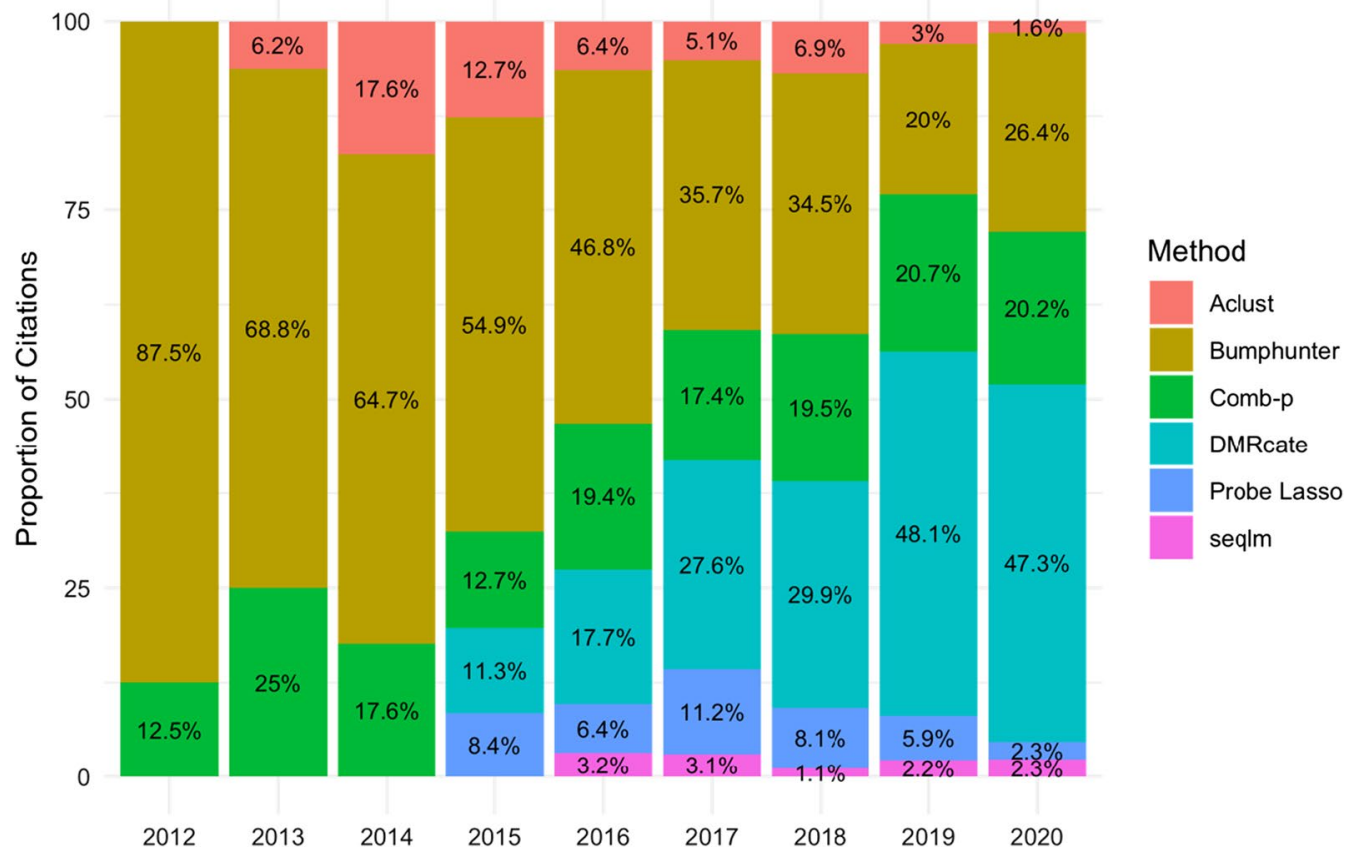

Fig. 7 Popularity of DMR identification tools. The proportion of PubMed publications by year using common DMR identification tools. Abbreviations: $\mathrm{DMR}=$ differentially methylated regions

and power in large and small effect sizes [73]. However, it is common for reviewers to request DMR identification with DMRcate if researchers use another tool. Therefore, if researchers identify DMRs with a novel tool, we recommend also using DMRcate for publication purposes.

DMR prioritisation paradigm DMR lists contain a range of information, including mean and maximum absolute delta beta (ADB). To identify DMRs with the largest biological effects, they can be ranked by either the mean or maximum ADB. DMPs with opposite directions of effect within a single DMR can reduce the size of the mean ADB and cause important biological effects to be missed. Therefore, we recommend ranking by maximum ADB. DMRs with an ADB below 2\% (0.02) should be interpreted with caution as it is difficult to discern whether these effects are caused by small, true biological effects or technical variation [6]. DMRs with an $\mathrm{ADB}>5 \%(0.05)$ can be classified as major DMRs and used for downstream analyses, as shown in past studies $[13,14,66]$. Biological processes driving the phenotype may be a cumulative effect of several DMRs with small ADB (effect). Therefore, we recommend using all DMRs with an $\mathrm{ADB}>0.02$ (2\%) for gene ontology analysis, which ideally require at least 50 genes to be informative. This paradigm is outlined in Fig. 8.

\section{Gene set enrichment analysis}

Gene set enrichment analysis (GSEA) determines whether an a priori list of genes is enriched for specific biological terms or pathways, and the association of these with the outcome of interest. GSEA can allow biologically relevant insights to be gleaned from DMPs and DMRs. While initially developed for gene expression studies, GSEA can be applied to gene lists obtained from EWASes. Due to widespread use in genomic research, many GSEA tools have been developed and comprehensively reviewed elsewhere. Here, we will discuss just some of those that are useful for analysis of gene sets identified from EWASes.

The ToppGene Suite is an online tool for conducting GSEA and candidate gene prioritisation using functional annotation and/or protein-protein interaction networks [77]. ToppGene is a quick and easy way to perform an initial exploration of gene sets as it interrogates 14 annotation categories to detect functional enrichment of a gene list. These include gene expression, protein functional domains, protein-protein interactions, transcription factor binding sites, microRNAs, gene ontology terms (molecular functions, cellular components and biological processes), pathways, human disease phenotypes, mouse phenotypes, drug-gene association and literature [77]. Statistical associations between genes and annotation categories are tested using hypergeometric tests [77]. 


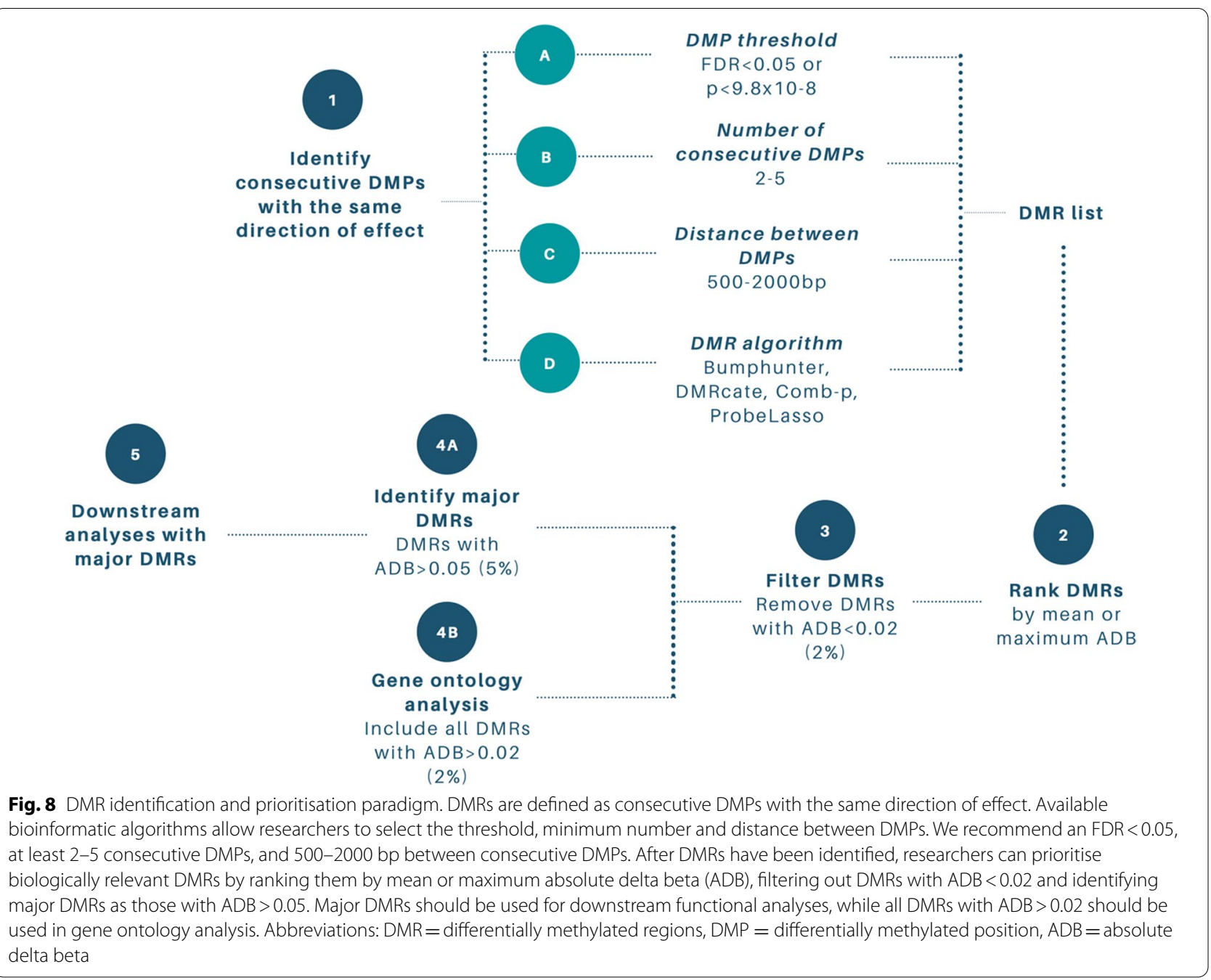

Notably, hypergeometric tests are unable to consider correlations between genes in the same gene set. ToppGene provides several significance thresholds for researchers to use: an unadjusted $p$ value, Benjamini-Hochberg FDR, Benjamini and Yekutieli FDR and Bonferroni FDR. As per differential methylation analysis, there is no standard significance threshold for GSEA. We recommend a Benjamini-Hochberg FDR threshold of 0.05 as it balances statistical stringency with exploration of biological results, to generate hypotheses.

For GSEA with ChAMP, a list of genes from DMP and/ or DMR analysis is required [3]. The Fisher exact test is the default method used by the function champ.GSEA(), but users can also select the ebGSEA or GOmeth [78] algorithm. Of these three algorithms, the Fisher exact test is the only one that fails to account for gene length bias; a phenomenon where longer genes are overrepresented in GSEA results, as they contain more CpGs than shorter genes [79]. Similarly, probe-number bias refers to the overrepresentation of genes in GSEA that contain more probes. The $e b G S E A$ algorithm applies an empirical Bayes method to a normalised beta-matrix and outcome of interest [80]. It overcomes gene length bias by identifying and ranking differentially methylated genes, rather than CpGs, before performing one-tailed Wilcoxon ranksum or known population median tests $[80,81]$. The GOmeth algorithm overcomes gene-length and probenumber bias by correcting analyses for the number of CpGs in a gene using the missMethyl package [78]. It also overcomes multi-gene bias, defined as a single CpG mapping to more than one gene, leading to false positive associations. It does this with fractional weighting in the Wallenius' non-central hypergeometric test, whereby the two genes that a CpG maps to each contribute a 0.5 weight to the intersection statistic of the test [78]. Genelength and probe-number bias are also present in DMR analysis, whereby DMRs are more likely to be called for genes that contain more probes. There is currently only 
one DMR GSEA tool that addresses these biases, GOregion. The GOregion function of the missMethyl package [78] uses the DMRcate output object to identify the CpGs in each DMR, before passing them to GOmeth. GOmeth has demonstrated higher accuracy and specificity than standard GSEA tools for methylation data, including ebGSEA [78].

\section{Protein-protein interaction analysis}

Protein-protein interactions (PPIs) are the basis of biological function that are affected by several factors including disease and therapeutics, and interact with molecules such as DNA [82]. PPI networks (PPINs) are mathematical representations of PPIs and can be used to understand the molecular drivers of disease states or identify potential therapeutic targets [82]. As per GSEA, a vast number of tools to identify PPINs from a gene list have been developed, some of which are discussed below. These tools utilise publicly available PPI data for which the reliability and level of annotation varies. Databases such as STRING [83] have tried to address this issue; however, it is recommended that researchers conduct PPIN analyses as an exploratory exercise, and interpret results with caution [83].

The EpiMod function in ChAMP uses the functional supervised algorithm of the $F E M$ package to identify functional epigenetic modules (FEMs). FEMs are gene modules with synchronised differential methylation and expression; integrating both methylation and expression data regardless of whether the data is matched [11, 84]. Using DMP association statistics, the FEM algorithm identifies PPI subnetworks in the protein interactome that have a significant number of genes associated with the study's outcome of interest [84].

The ToppGene application TopGeNet uses a list of genes to mine the protein interactome and identify genes directly or indirectly related to those on the gene list [77]. Genes that are identified are then ranked using PPIN analysis and functional annotations [77]. In PPIN analysis, genes are prioritised based on topological features of the network, quantified using PageRank with Priors, HITS with Priors and K-step Markov algorithms.

\section{Downstream analyses}

\section{Methylation risk scores}

The development and progression of complex diseases are polygenic. In recent years, polygenic risk scores (PRSs) have become a popular method to quantify cumulative risk of disease caused by changes with small effect sizes at multiple genes identified in GWASes. Similarly, methylation risk scores (MRSs) combine DMPs identified in EWASes into a meaningful indicator of risk at individual levels.
To construct a simple MRS, first identify DMPs associated with the trait of interest. Each DMP effect can be weighted based on their effect size. A simple MRS can be calculated for each sample as follows:

$$
\mathrm{MRS}_{j}=\sum w_{i} \beta_{i}
$$

where $w_{i}$ represents the weight assigned to DMP, while $\beta_{i}$ represents the methylation level (beta- or $M$ values) at DMP $i$ for individual $j$.

As per linkage disequilibrium in genetic studies, methylation can be highly correlated between probes (e.g. CPG islands located in promoter region of genes). Thus, removing highly correlated probes prior to MRS calculation will reduce the risk of the same signal being ascribed too much weight in the MRS. We recommend applying this filtering step prior to MRS calculation.

Simple MRSs, as well as more complex approaches, have been successfully implemented in the context of various complex diseases [85-87]. For guidance on constructing more complex MRSs, we recommend the recently published review by Hüls and Czamara [88].

\section{Methylation quantitative trait loci analysis}

Quantitative trait loci (QTL) are genomic loci influencing a quantifiable trait or phenotype [89]. The goal of QTL studies is to model the effect of genome variations on a quantifiable trait(s). QTL analysis can be used to map the relationship between methylation levels and genotype at a specific locus (methylation quantitative trait loci), and in turn determine how this relates to disease outcome (Fig. 9). Methylation quantitative trait loci (commonly abbreviated to methQTLs, metQTL or mQTLs) have been consistently identified across various diseases, populations and developmental stages [90]. There are several ways to identify methQTLs.

In a case-control cohort, one approach to identify mQTLs is to select DMPs associated with the phenotype and then select SNPs with minor allele frequency $(\mathrm{MAF}) \geq 0.05$ in the same genomic region [91]. Alternatively, researchers can select SNPs associated with the phenotype, and then select CpG sites in the same genomic region [92]. Either way, we recommend limiting the maximum window between CpG sites and SNPs, so only proximal or cis-methQTLs are identified. cismethQTLs refer to SNPs that are associated with CpGs within the same gene. The median window size for a cismethQTL is approximately $18 \mathrm{~kb}$ [93]; however, common thresholds range from 50 [92] to $100 \mathrm{~kb}$ [94]. After feature selection, statistical modelling is commonly used to identify how the methQTLs are associated with phenotype [90]. 


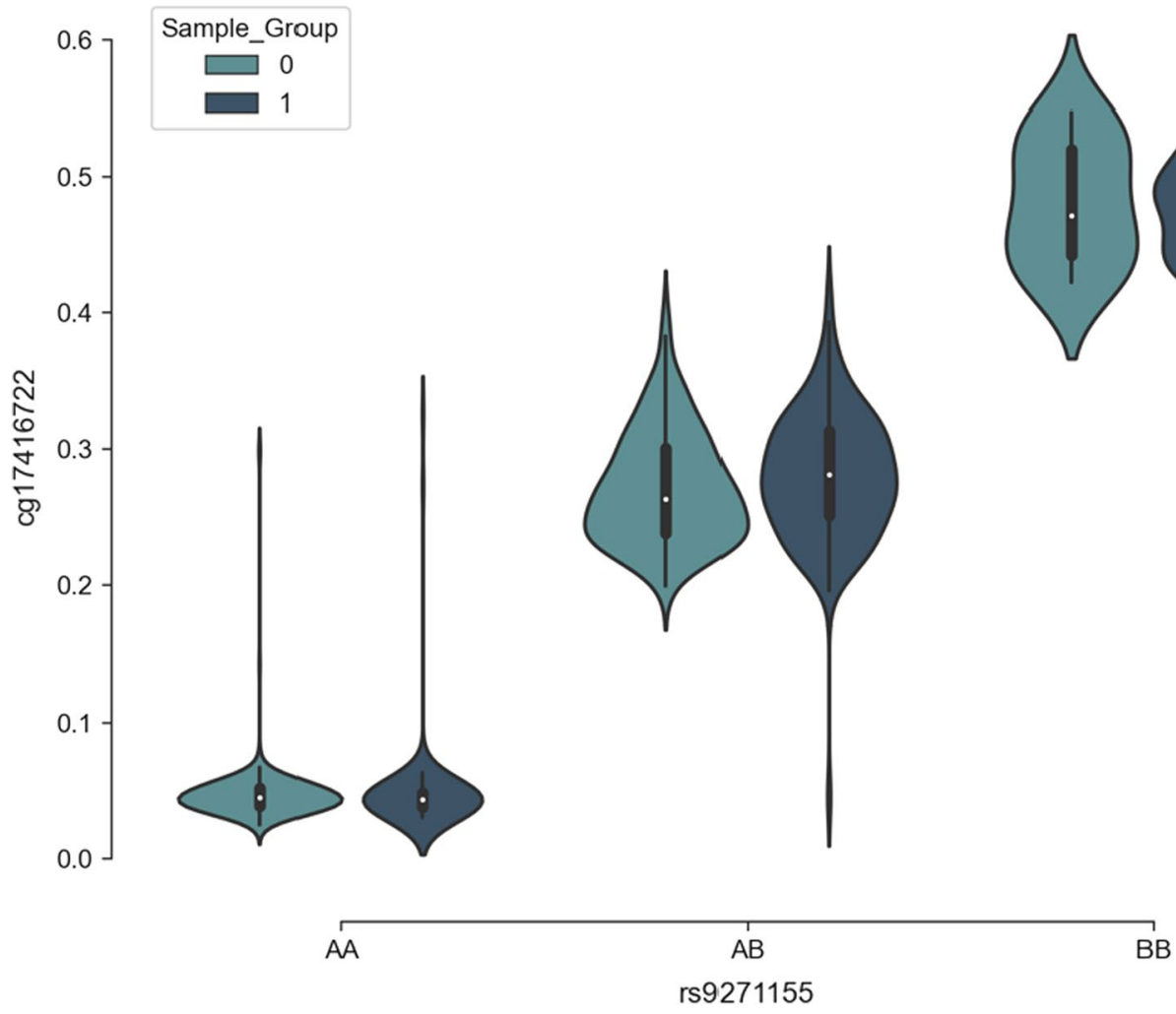

Fig. 9 methQTL at polymorphism rs9271155 based on sample group. Genotype at rs9271155 affects methylation level at CpG site cg17416722, whereby individuals with AA genotype at rs9271155 have low methylation levels at cg17416722, and individuals with BB genotype have high methylation levels. Individuals with a heterozygous genotype (AB) have intermediate methylation levels. From [unpublished data]. Abbreviations: methQTL = methylation quantitative trait locus

Plink [95] software is commonly used to conduct GWASes. The --assoc command also allows researchers to relate genomic data to quantitative traits, which can include methylation levels. Using this command, users can obtain statistical model results. Notably, plink will compare the linear regressions of sample groups and return summary statistics if researchers encode casecontrol labels as covariates.

There are packages available for more advanced methQTL calculations. For example, the R package GEM [96] can model the complex relationship between methylation levels, genotype, covariates and one environmental variable, which could be replaced for a phenotype variable. Further, it reduces the computational time of statistical modelling by using the matrix eQTL implementation, which involves unique data pre-processing and use of large matrix operations [97].

Another method for identifying methQTLs (and QTLs in general [98]) is RUV, which uses negative controls to identify and remove unwanted variation [60, 61]. It models the association between the log of CpG methylation (Y) and factors of interests $(\mathrm{X})$ while considering unwanted variation $(\mathrm{W})$.

The causal inference test (CIT) [99] has also been used to identify methQTLs. CIT evaluates four different conditions that help to identify the relationship between genotype, methylation levels and phenotype. Then, for each CpG-variant pair, the relationship is classified as either: (1) null, (2) independent, (3) independent/hidden variable, (4) causal, (5) causal/independent or (6) causal/hidden. CIT is mostly targeted at continuous phenotypes but can be modified to fit categorical phenotypes, including longitudinal study time points [100].

methQTLs examine the relationship between genotype and methylation. Since this relationship is diseaseagnostic and driven by genotype, it is possible to build large databases of methQTLs for researchers to leverage. The Genetics of DNA Methylation Consortium (mqtldb.godmc.org.uk), published in 2021, indexes over 270,000 methQTLs identified across 30,000 samples from over 30 cohorts [101]. As seen with eQTL databases [102], methQTL databases allow researchers 
to investigate the relationship between genotype and methylation in DMRs, in relation to a trait of interest.

\section{Estimating cell type proportions}

For EWASes performed using DNA from mixed cell samples, such as peripheral blood mononuclear cells (PBMCs), it is important to consider that methylation state can vary substantially by cell subtype. Failing to address this technical characteristic with deconvolution methods can lead to two main issues, namely spurious associations between CpGs and phenotypes due to variation in cell proportion, and/or missing potentially important cell-type specific DMPs. Several statistical methods have been developed to estimate the relative proportions of the major blood cell subtypes using methylation data derived from whole blood or PBMC samples. These algorithms can be classified either as "reference-based" [103], or "reference-free" [104, 105], depending on whether they use an a priori database of cell-type specific DNA methylation reference profiles to perform deconvolution. Reference-free methods are attractive because they can, in principle, be applied to any tissue; however, the reliability of the estimates has not been well established for many cell types. Furthermore, reference-free methods do not necessarily provide individual-level estimates of cell proportions, which offer flexibility for downstream association modelling. Therefore, reference-based methods are currently the most widely used in EWASes.

The first reference-based method developed was the so called "Houseman algorithm" [103], which remains the most cited algorithm to date. Of the 182 studies published in 2020 (available on PubMed) using cell type proportion algorithms, $92.3 \%$ used the Houseman algorithm. This algorithm estimates cell proportions using a variant of regression-based calibration known as linear constrained projection (LCP), whereby non-negativity and normalization constraints on cellular proportions are imposed based on observed cell-specific data.

More recently, Teschendorff et al. [106] developed a reference-based method that used a technique called robust partial correlations (RPC). Using empirical and simulated methylation datasets, they showed that the RPC method accurately estimated proportions of the major blood cell types, and outperformed LCP in terms of error of the estimates. Thus, for accurate estimation of blood cell subtype proportions, we recommend the tool EpiDISH, which is available as a Bioconductor package in the $\mathrm{R}$ statistical environment [107]. Individual-level proportion estimates derived from EpiDISH can then be included in association models as covariates, and in doing so, can help identify DMPs associated with phenotypes that are independent of (or adjusted for) cell subtype variation.

\section{Identifying cell-specific differential methylation from mixed cell data}

The methods available for identifying cell-specific differential methylation (CDM) using mixed cell data are less developed. This is mainly due to the statistical complexity of the problem, which involves interactive effects and lack of empirical data to validate statistical models. Fundamentally, identifying CDM relies on accurate cell type proportion estimation. These estimates can then be regressed against phenotypes to detect interactive effects between cell proportions and site-specific methylation signal, which indicates a CDM effect on the phenotype. Using this premise, Zheng et al. [108, 109] developed a tool called CellDMC. CellDMC takes the cell proportion estimates derived from EpiDISH [107] as input, and tests for interactive effects to indicate site-specific CDM signal. This method was validated using both empirical and simulated datasets, achieving $>90 \%$ sensitivity and specificity. The main issue with applying CellDMC is the power limitation. Zheng et al. showed that sample sizes of 100 cases and 100 controls will achieve approximately $80 \%$ power to detect CDM of $20 \%$ or more [109]. However, effect sizes are typically much less than this (sometimes $<5 \%$ ), and therefore, CDM signals may not be detected. Fortunately, the continued reduction in costs of methylation microarrays and the availability of large cohorts from the GWAS era mean that the detection of important CDM signals is becoming more feasible.

\section{Methylation age acceleration analysis}

Epigenetic age, or more specifically, methylation age, is a form of biological age calculated from methylation levels at CpG sites that are associated with chronological age. These CpGs are known as clock CpGs. Several algorithms, known as indices or clocks, that calculate methylation age using epigenome-wide methylation data have been created. They can be broadly categorised into chronological and biological indices. Chronological indices predict chronological age from methylation levels, while biological indices are a form of biological age that correlate with health, lifespan and clinical outcomes. Methylation age acceleration (MAA) is calculated as the residual term of regressing chronological age on methylation age, allowing researchers to compare MAA between groups or timepoints of interest.

\section{Chronological age indices}

The Horvath index (2013) [110] was the first index created and is currently the most widely cited (Fig. 10). It 

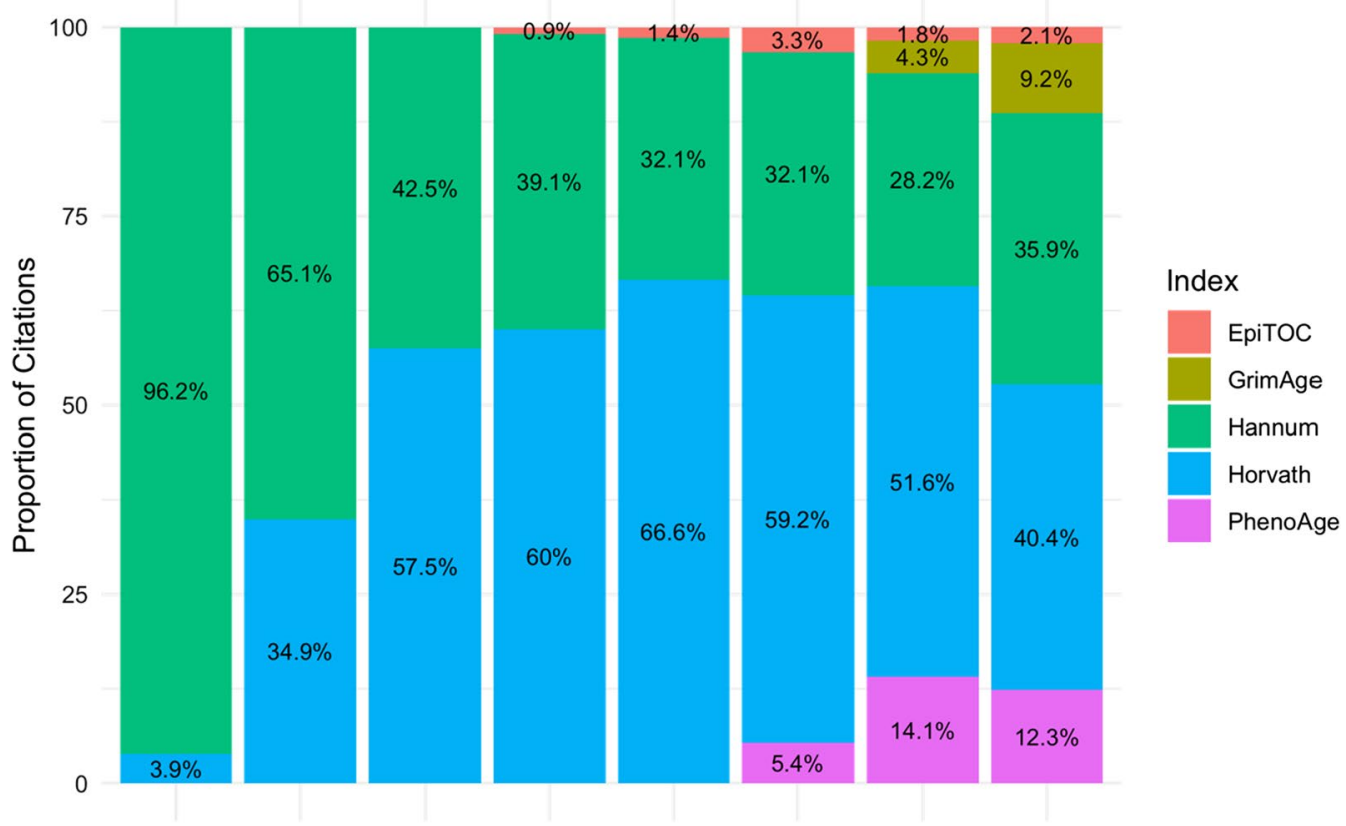

Fig. 10 Popularity of methylation age indices. The proportion of PubMed publications by year for DNA methylation age indices

uses methylation levels at 353 clock CpGs to predict chronological age within a five-year margin and a correlation of over 0.9 in different tissue types [110]. The strength of this index is the accurate prediction of chronological age in a range of tissues types [110]. However, tissue-specific methylation age acceleration, rather than systemic acceleration, likely occurs in many diseases. Thus, the pan-tissue nature of the Horvath index may limit its use in studies of disease-states. The Hannum index [111] was created to predict chronological age, accounting for four confounding factors gender, body mass index (BMI) and genotype [111]. It uses 71 clock CpGs to predict chronological age within 4.9 years and a correlation of 0.91 in whole blood [111]. These clock CpGs were mapped to genes associated with ageingrelated conditions, metabolism and obesity [111]; suggesting a biological relevance to these clock CpGs not seen with the Horvath index. MAA using the Horvath index is associated with all-cause morbidity and mortality. However, recent studies have shown that MAA calculated with the Horvath and Hannum indices are confounded by age-related changes in cell-type proportions [112]. The main limitation of these indices that they predict chronological age exceptionally accurately and consequently, cannot identify individuals of the same chronological age but different biological ages. As biological age is more closely associated with health or disease than chronological age, it can be argued that the Horvath and Hannum indices are unlikely to provide insights into an individual's risk of, and the mechanisms driving, disease and mortality.

\section{Biological age indices}

The PhenoAge index (2018) [113] was developed to more accurately predict lifespan and "healthspan" by including 513 CpGs that are associated with clinical differences between individuals of the same chronological age, as well as chronological age itself. The chosen CpGs are enriched in CpG islands and Polycomb group protein targets [113]. Methylation at Polycomb group protein targets promoter regions has previously been correlated with the number of stem cell divisions, related to the stem cell theory of ageing [114]. Studies have shown that a one-year increase in PhenoAge represents a $4.5 \%$ increased risk of all-cause mortality [113], and more generally, a higher PhenoAge predicts a shorter lifespan and more age-related comorbidities (i.e. shorter healthspan) [113]. A notable limitation of the PhenoAge index is its restriction to European cohorts as there is evidence of the index tagging ethnicity, as DNA methylation is a highly heritable trait, rather than health-related outcomes.

The GrimAge index (2019) [115] is the newest methylation age index, and predicts morbidity and mortality using surrogate DNA methylation biomarkers of demographic, clinical and lifestyle variables. The GrimAge index uses methylation levels at $1030 \mathrm{CpGs}$ to calculate 
methylation age. GrimAge can be adjusted for chronological age to obtain GrimAge Acceleration (AgeAccelGrim), which accurately predicts time to death, time to cancer and time to coronary heart disease [115]. AgeAccelGrim is further associated with T-cell senescence [116], age-related changes in blood cell proportions and leukocyte telomere length [115].

epiTOC (2016) [114] is an epigenetic mitotic clock that estimates the rate of stem cell divisions using methylation levels at 385 Polycomb group target (PCGT) promoter CpGs. As PCGT promoters are unmethylated in foetal tissue [117], age-associated hypermethylation and cumulative methylation aberrations resulting from cell divisions are used to estimate the rate of stem cell divisions [117]. The universally accelerated epiTOC rate in pre-cancerous and cancerous lesions, and epithelial tissue exposed to carcinogens, is concordant with existing knowledge of the association between cancer risk and the rate of stem cell divisions [118]. Thus, epiTOC is powerful in accurately estimating cancer risk using whole-blood methylation data [114]. The recently published epiTOC2 uses a similar method to directly estimate the number of stem cell divisions (rather than the rate), which can be used to differentiate cancer risk between tissue types [119].

For researchers to harness the accuracy of chronological age indices alongside the biological insight provided by biological age indices, we recommend calculating methylation age using a range of indices. However, the GrimAge clock deserves the most attention as this has been shown to be the single best performing index for assessing differential epigenetic ageing and predicting morbidity and mortality. In longitudinal studies, associating differences in chronological and methylation age trajectories with clinical outcomes may provide insight into disease mechanisms and risk factors. Therefore, we recommend methyAge function in the Bioconductor package ENmix, to calculate methylation age using the Horvath [110], Hannum [111] and PhenoAge [113]

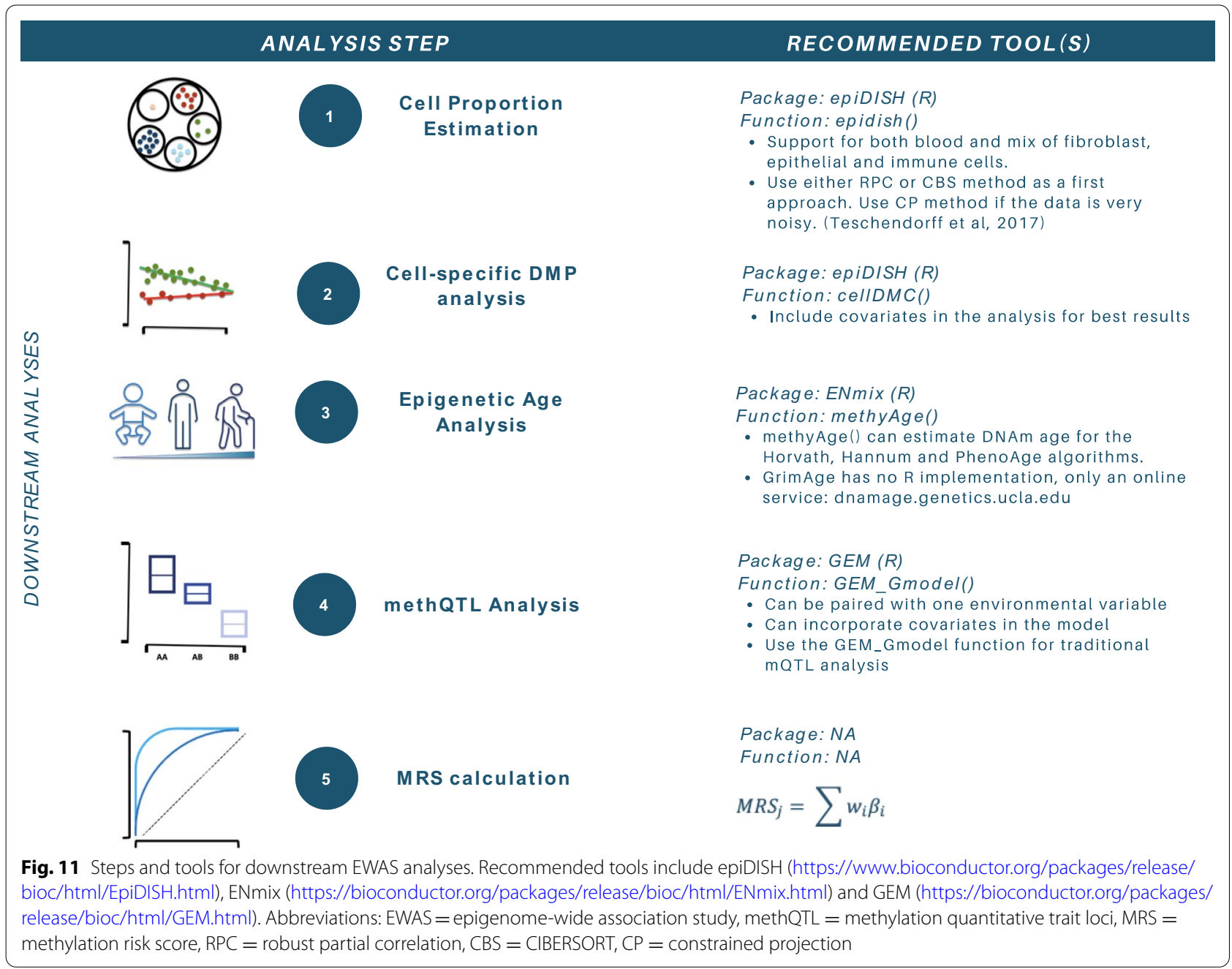


indices. GrimAge can be calculated using an online DNA methylation age calculator [120].

Useful tools for the downstream analyses discussed above are recapitulated in Fig. 11.

\section{Epigenome-wide association study databases}

The number of published EWASes and associated methylation data has risen exponentially since 2009 (Fig. 1) and will continue to do so as the cost of microarrays fall. To harness this magnitude of data for scientific purposes, researchers have created databases of EWAS data. Such databases can be categorised into Deposition, Integration or Association databases (Fig. 12). Deposition databases contain raw methylation data and metadata, Integration databases contain normalised data and metadata, while Association databases contain normalised data, metadata and published associations at the CpG level.

Deposition databases, such as gene express omnibus (GEO) [121], primarily serve as data archives, as methylation data is not normalised or integrated across datasets, tissue types and diseases [121]. Integration databases, such as EWAS Datahub [122], have built on this by building a pipeline that performs data normalisation, batch effect correction and data standardisation across datasets [123]. Association databases, such as EWAS Atlas [124], further build on integration databases by including EWAS associations, manually curated from published literature [125]. EWAS databases provide a useful resource for researchers to perform analyses when laboratory resources are limited, or to validate preliminary findings. Further to this, they are valuable for untangling the molecular mechanisms underpinning methylation-trait associations, aiding in the development of diagnostic, prognostic and therapeutic tools [126].

Researchers should note that differential methylation exists among racial/ethnic groups [127, 128]. In addition, to epigenetic drift, these differences will reflect differences in both genetic and environmental factors. Of the publications reported in the EWAS Atlas, 51\% represent from European cohorts, 19\% African and 20\% Asian cohorts [125]. Therefore, researchers should be mindful of the potential for confounding of results due to ethnic differences. Steps to guard against this include adding genomic structure components in the statistical modelling.

\section{Conclusion and future directions}

Blood cell-based EWASes are useful experimental designs for identifying cell-type independent or celltype specific methylation levels associated with an outcome trait of interest. The reducing cost of methylation microarrays and the advance of associated bioinformatics toolkits are aiding in the discovery of epigenetic factors related to disease outcomes. However, translating these differences into clinically useful findings, including prognostic biomarkers and therapeutic targets in the epigenome, has often been restricted by factors such as inappropriate or inadequate statistical analysis methods,

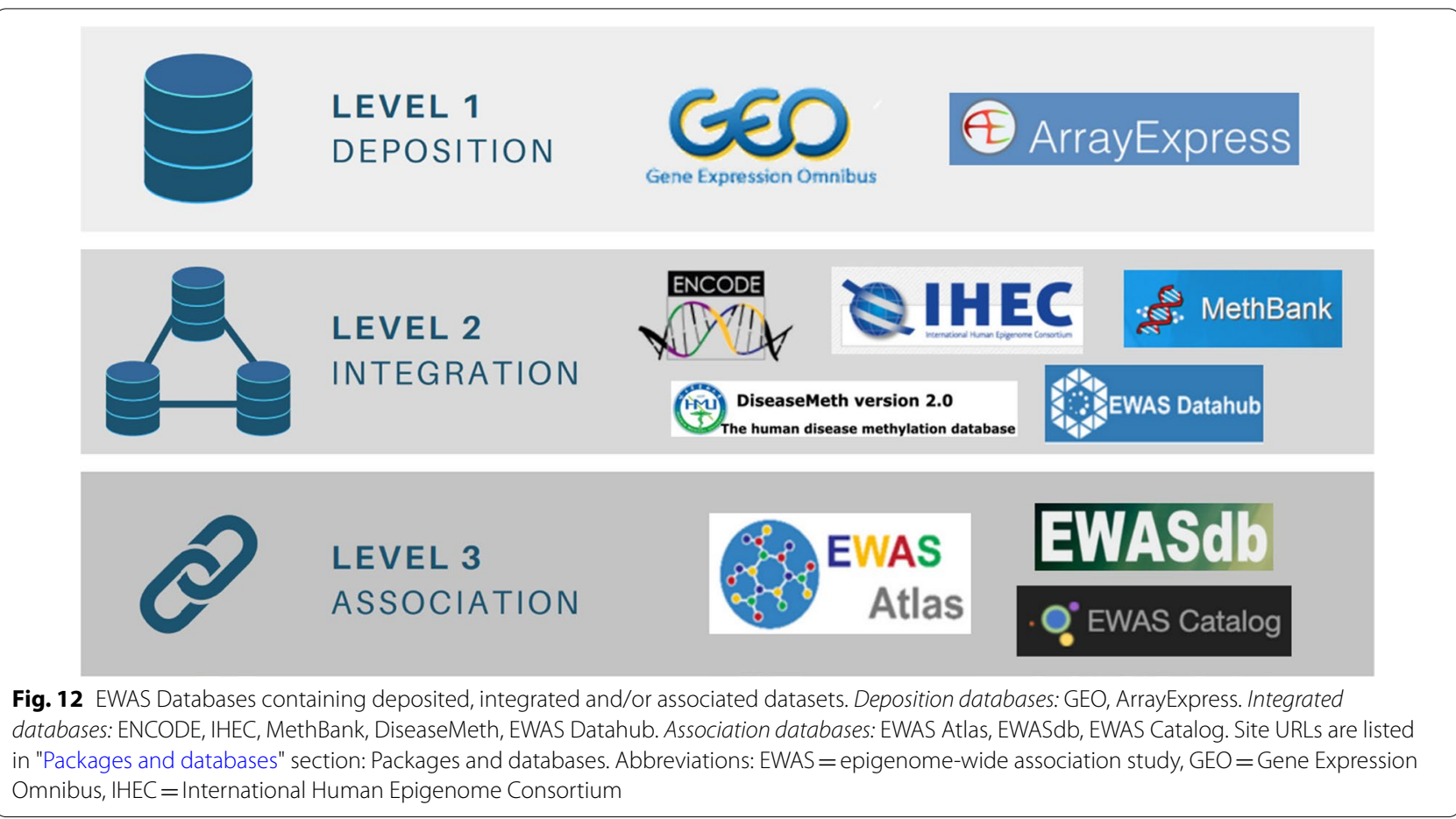


insufficiently powered sample sizes, non-validated findings and an inability to establish causality. Here, we provide up-to-date recommendations for maximising the value of EWASes based on these factors. We acknowledge that our commentary is restricted to blood-based EWASes. Therefore, extrapolation of our recommendations to EWASes of other cell and tissue types should be applied with caution since there are substantive epigenetic differences. Similarly, we focus on one epigenetic mechanism, DNA methylation, without discussing histone modification and post-transcriptional regulation. These epigenetic mechanisms work together to regulate gene expression, and therefore, the study of one mechanism in isolation will limit biological understanding and the clinical relevance of results. Methods to study histone modification and post-transcriptional regulation have been reviewed previously $[129,130]$.

Lastly, whole-genome bisulphite sequencing (WGBS) is fast becoming a viable option for studying DNA methylation, due to recent advances in sequencing technologies. Compared to microarrays, WGBS measures methylation at a higher density of $\mathrm{CpG}$ sites and detects non-CpG methylation [131]. Reduced representation bisulphite sequencing (RRBS) enriches and captures DNA fragments in CpG-rich regions using restriction enzymes. The costs of RRBS and microarray-based methylation studies are comparable and affordable as they continue to fall in cost, while WGBS remains prohibitively expensive-particularly in studies that require many samples. Compared to RRBS, microarrays have more consistent genome-coverage and methylation level estimations, making them a better choice for EWASes [132]. However, RRBS is more flexible and can be used to interrogate loci that are not covered by microarrays. Third-generation sequencing technologies, such as Oxford Nanopore Sequencing (ONS), conduct long-read DNA and methylation sequencing simultaneously. Advantages of ONS include little sample preparation as bisulphite conversion is not required as with microarrays. Furthermore, ONS measures $5 \mathrm{mC}, 5 \mathrm{hmC}, 6 \mathrm{~mA}$ methylation, while microarrays measure $5 \mathrm{mC}$ only.

Nevertheless, Illumina microarrays are currently $12-15 \%$ more accurate than Oxford nanopore sequencing [133], and therefore remain the most widely used sequencing technology for EWASes.

In this review, we have critically compared multiple aspects of EWAS study design and bioinformatic analysis, including numerous tools yet to be reviewed, to provide recommendations to researchers new to conducting blood cell-based EWASes.

\section{Packages and databases}

\begin{tabular}{|c|c|}
\hline ChAMP & $\begin{array}{l}\text { https://bioconductor.org/packages/ } \\
\text { release/bioc/html/ChAMP.html }\end{array}$ \\
\hline Minfi & $\begin{array}{l}\text { https://bioconductor.org/packages/ } \\
\text { release/bioc/html/minfi.html }\end{array}$ \\
\hline Limma & $\begin{array}{l}\text { https://bioconductor.org/packages/ } \\
\text { release/bioc/html/limma.html }\end{array}$ \\
\hline ComBat & $\begin{array}{l}\text { https://rdrrio/bioc/sva/man/ } \\
\text { ComBat.html }\end{array}$ \\
\hline Bumphunter & $\begin{array}{l}\text { https://www.bioconductor.org/ } \\
\text { packages/release/bioc/html/ } \\
\text { bumphunter.html }\end{array}$ \\
\hline DMRcate & $\begin{array}{l}\text { https://bioconductor.org/packages/ } \\
\text { release/bioc/html/DMRcate.html }\end{array}$ \\
\hline Comb-p & $\begin{array}{l}\text { https://rdrr.io/bioc/ENmix/man/ } \\
\text { combp.html }\end{array}$ \\
\hline PLINK & https://zzz.bwh.harvard.edu/plink/ \\
\hline GEM & $\begin{array}{l}\text { https://bioconductor.org/packages/ } \\
\text { release/bioc/html/GEM.html }\end{array}$ \\
\hline epiDISH & $\begin{array}{l}\text { https://www.bioconductor.org/ } \\
\text { packages/release/bioc/html/EpiDI } \\
\text { SH.html }\end{array}$ \\
\hline ENmix & $\begin{array}{l}\text { https://bioconductor.org/packages/ } \\
\text { release/bioc/html/ENmix.html }\end{array}$ \\
\hline missMethyl & $\begin{array}{l}\text { http://bioconductor.org/packages/ } \\
\text { release/bioc/html/missMethyl.html }\end{array}$ \\
\hline ToppGene & https://toppgene.cchmc.org/ \\
\hline FEM & $\begin{array}{l}\text { http://bioconductor.riken.jp/packa } \\
\text { ges/3.7/bioc/html/FEM.html }\end{array}$ \\
\hline GEO & https://www.ncbi.nlm.nih.gov/geo/ \\
\hline ArrayExpress & https://www.ebi.ac.uk/arrayexpress/ \\
\hline ENCODE & https://www.encodeproject.org/ \\
\hline IHEC & https://ihec-epigenomes.org/ \\
\hline MethBank & http://bigd.big.ac.cn/methbank \\
\hline DiseaseMeth & $\begin{array}{l}\text { http://bio-bigdata.hrbmu.edu.cn/ } \\
\text { diseasemeth }\end{array}$ \\
\hline EWAS Datahub & $\begin{array}{l}\text { https://bigd.big.ac.cn/ewas/datah } \\
\text { ub }\end{array}$ \\
\hline EWAS Atlas & http://bigd.big.ac.cn/ewas \\
\hline EWASdb & http://www.bioapp.org/ewasdb/ \\
\hline EWAS Catalog & http://www.ewascatalog.org/ \\
\hline $\begin{array}{l}\text { The Genetics of DNA Methylation } \\
\text { Consortium }\end{array}$ & http://mqtldb.godmc.org.uk \\
\hline
\end{tabular}

\section{Abbreviations}

ADB:: Absolute delta beta; BMIQ:: Beta-mixture quantile normalisation; $C D M::$ Cell-specific differential methylation; ChAMP.: Chip analysis methylation pipeline; CIT:: Causal inference test; CpG:: Cytosine-phosphate-guanine; DMP:: Differentially methylated position or probe; DMR:: Differentially methylated region; DZ:: Dizygotic; EPIC:: Illumina HumanMethylation850 microarray; eQTL: Expression quantitative trait loci; EWAS:: Epigenome-wide association study; FDR:: False-discovery rate; FEM:: Functional epigenetic modules; Funnorm:: Functional normalisation; GSEA:: Gene set enrichment analysis; MAA:: Methylation age acceleration; MAF:: Minor allele frequency; methQTL:: Methylation 
quantitative trait loci; MHC:: Major histocompatibility complex; MZ:: Monozygotic; PBMCs:: Peripheral blood mononuclear cells; PPIN:: Protein-protein interaction; QC:: Quality control; RRBS:: Reduced representation bisulphite sequencing; RUV:: Remove unwanted variation; SNPs:: Single-nucleotide polymorphisms; SSnoob:: Single-sample noob; SVD:: Singular value decomposition; SWAN:: Subset-quantile within microarray normalisation; WGBS:: Whole-genome bisulphite sequencing; 27k:: Illumina HumanMethylation27 microarray; 450k:: Illumina HumanMethylation450 microarray.

\section{Acknowledgements}

None.

\section{Authors' contributions}

RL conceptualised the paper. MPC, AX and RL wrote the manuscript. All authors read and provided feedback on manuscript drafts and approved the final manuscript.

\section{Funding}

None.

\section{Availability of data and materials}

Not applicable.

\section{Declarations}

\section{Ethics approval and consent to participate}

Not applicable.

\section{Consent for publication}

Not applicable.

\section{Competing interests}

The authors declare that they have no competing interests.

\section{Author details}

${ }^{1}$ Department of Neuroscience, Central Clinical School, Monash University, Melbourne, Australia. ${ }^{2}$ Centre for Information Based Medicine, Hunter Medical Research Institute, Newcastle, Australia. ${ }^{3}$ School of Biomedical Sciences and Pharmacy, University of Newcastle, Newcastle, Australia. ${ }^{4}$ Department of Neurology, Division of Medicine, John Hunter Hospital, Newcastle, Australia. ${ }^{5}$ Division of Molecular Medicine, New South Wales Health Pathology North, Newcastle, Australia. ${ }^{6}$ Department of Neurology, Alfred Health, Melbourne, Australia. ${ }^{7}$ Centre for Genomics and Personalised Health, School of Biomedical Sciences, Queensland University of Technology, Brisbane, Australia.

Received: 25 May 2021 Accepted: 19 November 2021

\section{Published online: 04 December 2021}

\section{References}

1. Zheleznyakova GY, Piket E, Marabita F, et al. Epigenetic research in multiple sclerosis: progress, challenges, and opportunities. Physiol Genomics. 2017;49(9):447-61.

2. Li X, Xiao B, Chen X-S. DNA methylation: a new player in multiple sclerosis. Mol Neurobiol. 2017;54(6):4049-59.

3. Hedrich CM, Mäbert K, Rauen T, Tsokos GC. DNA methylation in systemic lupus erythematosus. Epigenomics. 2017;9(4):505-25.

4. Guo S, Xu L, Chang C, et al. Epigenetic regulation mediated by methylation in the pathogenesis and precision medicine of rheumatoid arthritis. Front Genet. 2020;11:811.

5. Bibikova M, Le J, Barnes B, et al. Genome-wide DNA methylation profiling using Infinium assay. Epigenomics. 2009;1(1):177-200.

6. Bibikova M, Barnes B, Tsan C, et al. High density DNA methylation array with single CpG site resolution. Genomics. 2011;98(4):288-95.

7. Moran S, Arribas C, Esteller M. Validation of a DNA methylation microarray for $850,000 \mathrm{CpG}$ sites of the human genome enriched in enhancer sequences. Epigenomics. 2016;8(3):389-99.
8. Aryee MJ, Jaffe AE, Corrada-Bravo H, et al. Minfi: a flexible and comprehensive bioconductor package for the analysis of Infinium DNA methylation microarrays. Bioinformatics. 2014;30(10):1363-9.

9. Morris TJ, Butcher LM, Feber A, et al. ChAMP: 450k chip analysis methylation pipeline. Bioinformatics. 2014;30(3):428-30.

10. Fortin J-P, Triche TJ, Hansen KD. Preprocessing, normalization and integration of the Illumina HumanMethylationEPIC array with minfi. Bioinformatics. 2017;33(4):558-60.

11. Tian Y, Morris TJ, Webster AP, et al. ChAMP: updated methylation analysis pipeline for Illumina BeadChips. Bioinformatics. 2017;33(24):3982-4.

12. Tan Q, Christiansen L, von Bornemann Hjelmborg J, Christensen K. Twin methodology in epigenetic studies. J Exp Biol. 2015;218(1):134-9.

13. Maltby VE, Graves MC, Lea RA, et al. Genome-wide DNA methylation profiling of CD8+ T cells shows a distinct epigenetic signature to CD4+ T cells in multiple sclerosis patients. Clin Epigenet. 2015;7(1):118.

14. Maltby VE, Lea RA, Sanders KA, et al. Differential methylation at MHC in CD4+ T cells is associated with multiple sclerosis independently of HLA-DRB1. Clin Epigenet. 2017;9(1):71.

15. Maltby VE, Lea RA, Graves MC, et al. Genome-wide DNA methylation changes in CD19+ B cells from relapsing-remitting multiple sclerosis patients. Sci Rep. 2018;8(1):17418.

16. Kulakova OG, Kabilov MR, et al. Whole-genome DNA methylation analysis of peripheral blood mononuclear cells in multiple sclerosis patients with different disease courses. Acta Nat. 2016:8(3):103-10.

17. Rakyan VK, Down TA, Balding DJ, Beck S. Epigenome-wide association studies for common human diseases. Nat Rev Genet. 2011;12(8):529-41.

18. Jamieson E, Korologou-Linden R, Wootton RE, et al. Smoking, DNA methylation, and lung function: a mendelian randomization analysis to investigate causal pathways. Am J Hum Genet. 2020;106(3):315-26.

19. Martino DJ, Tulic MK, Gordon L, et al. Evidence for age-related and individual-specific changes in DNA methylation profile of mononuclear cells during early immune development in humans. Epigenetics. 2011;6(9):1085-94.

20. Wang D, Liu X, Zhou Y, et al. Individual variation and longitudinal pattern of genome-wide DNA methylation from birth to the first two years of life. Epigenetics. 2012;7(6):594-605.

21. Acevedo N, Reinius LE, Vitezic M, et al. Age-associated DNA methylation changes in immune genes, histone modifiers and chromatin remodeling factors within 5 years after birth in human blood leukocytes. Clin Epigenet. 2015;7(1):34.

22. Urdinguio RG, Torró MI, Bayón GF, et al. Longitudinal study of DNA methylation during the first 5 years of life. J Transl Med. 2016;14(1):160.

23. Pérez RF, Santamarina P, Tejedor JR, et al. Longitudinal genome-wide DNA methylation analysis uncovers persistent early-life DNA methylation changes. J Transl Med. 2019;17(1):15.

24. Herbstman JB, Wang S, Perera FP, et al. Predictors and consequences of global DNA methylation in cord blood and at three years. PLOS ONE. 2013:8(9):e72824.

25. Torow N, Hornef MW. The neonatal window of opportunity: setting the stage for life-long host-microbial interaction and immune homeostasis. J Immunol Baltim Md. 2017;198(2):557-63.

26. Bjornsson HT, Sigurdsson MI, Fallin MD, et al. Intra-individual change in DNA methylation over time with familial clustering. JAMA J Am Med Assoc. 2008;299(24):2877-83.

27. Bollati V, Schwartz J, Wright $R$, et al. Decline in genomic DNA methylation through aging in a cohort of elderly subjects. Mech Ageing Dev. 2009;130(4):234-9.

28. Talens RP, Christensen $\mathrm{K}$, Putter $\mathrm{H}$, et al. Epigenetic variation during the adult lifespan: cross-sectional and longitudinal data on monozygotic twin pairs. Aging Cell. 2012;11(4):694-703.

29. Reynolds CA, Tan Q, Munoz E, et al. A decade of epigenetic change in aging twins: genetic and environmental contributions to longitudinal DNA methylation. Aging Cell. 2020;19(8):e13197.

30. Wang $Y$, Karlsson R, Lampa E, et al. Epigenetic influences on aging: a longitudinal genome-wide methylation study in old Swedish twins. Epigenetics. 2018;13(9):975-87.

31. Tan Q, Heijmans BT, von Bornemann Hjelmborg J, et al. Epigenetic drift in the aging genome: a ten-year follow-up in an elderly twin cohort. Int J Epidemiol. 2016;45:1146-58 
32. Gutierrez-Arcelus M, Ongen H, Lappalainen T, et al. Tissue-specific effects of genetic and epigenetic variation on gene regulation and splicing. PLoS Genet. 2015;11(1):e1004958.

33. Mansell G, Gorrie-Stone TJ, Bao Y, et al. Guidance for DNA methylation studies: statistical insights from the Illumina EPIC array. BMC Genomics. 2019;20(1):366.

34. Tsai P-C, Bell JT. Power and sample size estimation for epigenome-wide association scans to detect differential DNA methylation. Int J Epidemiol. 2015;44(4):1429-41.

35. Saffari A, Silver MJ, Zavattari P, et al. Estimation of a significance threshold for epigenome-wide association studies. Genet Epidemiol. 2018;42(1):20-33.

36. Henderson-Smith A, Fisch KM, Hua J, et al. DNA methylation changes associated with Parkinson's disease progression: outcomes from the first longitudinal genome-wide methylation analysis in blood. Epigenetics. 2019;14(4):365-82

37. Johnson RK, Vanderlinden LA, Dong F, et al. Longitudinal DNA methylation differences precede type 1 diabetes. Sci Rep. 2020;10(1):3721.

38. R Core Team. R: A language and environment for statistical computing. R Foundation for Statistical Computing; 2020

39. Chen $Y$, Lemire M, Choufani S, et al. Discovery of cross-reactive probes and polymorphic CpGs in the Illumina Infinium HumanMethylation450 microarray. Epigenetics. 2013;8(2):203-9.

40. Nordlund J, Bäcklin CL, Wahlberg P, et al. Genome-wide signatures of differential DNA methylation in pediatric acute lymphoblastic leukemia. Genome Biol. 2013;14(9):r105.

41. Pidsley R, Zotenko E, Peters TJ, et al. Critical evaluation of the Illumina MethylationEPIC BeadChip microarray for whole-genome DNA methylation profiling. Genome Biol. 2016;17(1):208.

42. Du P, Zhang X, Huang C-C, et al. Comparison of Beta-value and M-value methods for quantifying methylation levels by microarray analysis. BMC Bioinform. 2010;11:587.

43. Teschendorff AE, Marabita F, Lechner M, et al. A beta-mixture quantile normalization method for correcting probe design bias in illumina infinium 450 k DNA methylation data. Bioinformatics. 2013:29(2):189-96.

44. Maksimovic J, Gordon L, Oshlack A. SWAN: subset-quantile within array normalization for illumina infinium HumanMethylation450 BeadChips. Genome Biol. 2012;13(6):R44.

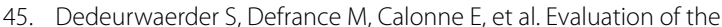
infinium methylation 450K technology. Epigenomics. 2011;3(6):771-84.

46. Fortin J-P, Labbe A, Lemire $M$, et al. Functional normalization of $450 \mathrm{k}$ methylation array data improves replication in large cancer studies. Genome Biol. 2014;15(11):1-17.

47. Wang T, Guan W, Lin J, et al. A systematic study of normalization methods for Infinium 450K methylation data using whole-genome bisulfite sequencing data. Epigenetics. 2015;10(7):662-9.

48. Dedeurwaerder S, Defrance $M$, Bizet $M$, et al. A comprehensive overview of Infinium HumanMethylation450 data processing. Brief Bioinform. 2014;15(6):929-41.

49. Marabita F, Almgren M, Lindholm ME, et al. An evaluation of analysis pipelines for DNA methylation profiling using the Illumina HumanMethylation450 BeadChip platform. Epigenetics. 2013;8(3):333-46.

50. Wu MC, Joubert BR, Kuan P, et al. A systematic assessment of normalization approaches for the Infinium 450K methylation platform. Epigenetics. 2014;9(2):318-29.

51. van Rooij J, Mandaviya PR, Claringbould A, et al. Evaluation of commonly used analysis strategies for epigenome- and transcriptome-wide association studies through replication of large-scale population studies. Genome Biol. 2019;20:1-14.

52. Price EM, Robinson WP. Adjusting for batch effects in DNA methylation microarray data, a lesson learned. Front Genet. 2018;9:83.

53. Buhule OD, Minster RL, Hawley NL, et al. Stratified randomization controls better for batch effects in 450K methylation analysis: a cautionary tale. Front Genet. 2014. https://doi.org/10.3389/fgene.2014.00354/full.

54. Nygaard V, Rødland EA, Hovig E. Methods that remove batch effects while retaining group differences may lead to exaggerated confidence in downstream analyses. Biostat Oxf Engl. 2016;17(1):29-39.

55. Zindler T, Frieling $H$, Neyazi A, et al. Simulating ComBat: how batch correction can lead to the systematic introduction of false positive results in DNA methylation microarray studies. BMC Bioinform. 2020;21:1-15.
56. Müller C, Schillert A, Röthemeier C, et al. Removing batch effects from longitudinal gene expression-quantile normalization plus ComBat as best approach for microarray transcriptome data. PLoS ONE. 2016;11(6):e0156594.

57. Fortin J-P, Parker D, Tunç B, et al. Harmonization of multi-site diffusion tensor imaging data. Neuroimage. 2017;161:149-70.

58. Yu M, Linn KA, Cook PA, et al. Statistical harmonization corrects site effects in functional connectivity measurements from multi-site fMRI data. Hum Brain Mapp. 2018:39(11):4213-27.

59. Jones SA, Morales AM, Holley AL, et al. Default mode network connectivity is related to pain frequency and intensity in adolescents. Neurolmage Clin. 2020;27:102326.

60. Gagnon-Bartsch JA, Speed TP. Using control genes to correct for unwanted variation in microarray data. Biostat Oxf Engl. 2012;13(3):539-52.

61. Removing Unwanted Variation from High Dimensional Data with Negative Controls | Department of Statistics. [cited 2021 Jan 20]. https://stati stics.berkeley.edu/tech-reports/820.

62. Ritchie ME, Phipson B, Wu D, et al. limma powers differential expression analyses for RNA-sequencing and microarray studies. Nucleic Acids Res. 2015;43(7):e47-e47

63. Benjamini Y, Hochberg Y. Controlling the false discovery rate: a practical and powerful approach to multiple testing. J R Stat Soc Ser B Methodol. 1995;57(1):289-300

64. Gerring ZF, McRae AF, Montgomery GW, Nyholt DR. Genome-wide DNA methylation profiling in whole blood reveals epigenetic signatures associated with migraine. BMC Genomics. 2018:19:69.

65. Jaffe $A E$, Murakami $P$, Lee $H$, et al. Bump hunting to identify differentially methylated regions in epigenetic epidemiology studies. Int J Epidemiol. 2012;41(1):200-9.

66. Maltby VE, Lea RA, Ribbons KA, et al. DNA methylation changes in CD4+ T cells isolated from multiple sclerosis patients on dimethyl fumarate. Mult Scler J Exp Transl Clin. 2018;4(3):2055217318787826.

67. Spindola LM, Santoro ML, Pan PM, et al. Detecting multiple differentially methylated CpG sites and regions related to dimensional psychopathology in youths. Clin Epigenet. 2019;11(1):146.

68. Eze IC, Jeong A, Schaffner $\mathrm{E}$, et al. Genome-wide DNA methylation in peripheral blood and long-term exposure to source-specific transportation noise and air pollution: the SAPALDIA study. Environ Health Perspect. 2020;128(6):67003.

69. Nilsson EE, Thorson JLM, Ben Maamar M, et al. Epigenome-wide association study (EWAS) for potential transgenerational disease epigenetic biomarkers in sperm following ancestral exposure to the pesticide methoxychlor. Environ Epigenet. 2020;6(1):dvaa020.

70. Sherwood WB, Kothalawala DM, Kadalayil L, et al. Epigenome-wide association study reveals duration of breastfeeding is associated with epigenetic differences in children. Int J Environ Res Public Health. 2020;17(10):E3569.

71. Li QS, Sun Y, Wang T. Epigenome-wide association study of Alzheimer's disease replicates 22 differentially methylated positions and 30 differentially methylated regions. Clin Epigenet. 2020;12(1):149.

72. Abeni E, Salvi A, Marchina E, et al. Sorafenib induces variations of the DNA methylome in HA22T/NGH human hepatocellular carcinomaderived cells. Int J Oncol. 2017;51(1):128-44.

73. Mallik S, Odom GJ, Gao Z, et al. An evaluation of supervised methods for identifying differentially methylated regions in Illumina methylation arrays. Brief Bioinform. 2019;20(6):2224-35.

74. Peters TJ, Buckley MJ, Statham AL, et al. De novo identification of differentially methylated regions in the human genome. Epigenet Chromatin. 2015;8:6.

75. Pedersen BS, Schwartz DA, Yang IV, Kechris KJ. Comb-p: software for combining, analyzing, grouping and correcting spatially correlated $p$ values. Bioinform Oxf Engl. 2012;28(22):2986-8.

76. Butcher LM, Beck S. Probe Lasso: a novel method to rope in differentially methylated regions with 450K DNA methylation data. Methods San Diego Calif. 2015;72:21-8.

77. Chen J, Bardes EE, Aronow BJ, Jegga AG. ToppGene suite for gene list enrichment analysis and candidate gene prioritization. Nucleic Acids Res. 2009;37(Web Server issue):W305-11. 
78. Maksimovic J, Oshlack A, Phipson B. Gene set enrichment analysis for genome-wide DNA methylation data. Bioinformatics. 2020. https://doi. org/10.1101/2020.08.24.265702.

79. Oshlack A, Wakefield MJ. Transcript length bias in RNA-seq data confounds systems biology. Biol Direct. 2009;4:14.

80. Dong D, Tian Y, Zheng SC, Teschendorff AE. ebGSEA: an improved gene set enrichment analysis method for epigenome-wide-association studies. Bioinformatics. 2019;35(18):3514-6.

81. Parks MM. An exact test for comparing a fixed quantitative property between gene sets. Bioinform Oxf Engl. 2018;34(6):971-7.

82. Safari-Alighiarloo N, Taghizadeh M, Rezaei-Tavirani M, et al. Proteinprotein interaction networks (PPI) and complex diseases. Gastroenterol Hepatol Bed Bench. 2014;7(1):17-31.

83. Szklarczyk D, Gable AL, Lyon D, et al. STRING v11: protein-protein association networks with increased coverage, supporting functional discovery in genome-wide experimental datasets. Nucleic Acids Res. 2019;47(Database issue):D607-13.

84. Jiao Y, Widschwendter M, Teschendorff AE. A systems-level integrative framework for genome-wide DNA methylation and gene expression data identifies differential gene expression modules under epigenetic control. Bioinform Oxf Engl. 2014;30(16):2360-6.

85. Odintsova V, Rebattu $\bigvee$, Hagenbeek FA, et al. Predicting complex traits and exposures from polygenic scores and blood and buccal DNA methylation profiles. Front Psychiatry. 2021;12:688464.

86. Onwuka JU, Li D, Liu Y, et al. A panel of DNA methylation signature from peripheral blood may predict colorectal cancer susceptibility. BMC Cancer. 2020;20(1):692.

87. Westerman K, Fernández-Sanlés A, Patil P, et al. Epigenomic assessment of cardiovascular disease risk and interactions with traditional risk metrics. J Am Heart Assoc. 2020;9(8):e015299.

88. Hüls A, Czamara D. Methodological challenges in constructing DNA methylation risk scores. Epigenetics. 2020;15(1-2):1-11.

89. Abiola O, Angel JM, Avner P, et al. The nature and identification of quantitative trait loci: a community's view. Nat Rev Genet. 2003;4(11):911-6.

90. Smith AK, Kilaru V, Kocak M, et al. Methylation quantitative trait loci (meQTLs) are consistently detected across ancestry, developmental stage, and tissue type. BMC Genomics. 2014;15:145.

91. Zhou F, Shen C, Xu J, et al. Epigenome-wide association data implicates DNA methylation-mediated genetic risk in psoriasis. Clin Epigenet. 2016;8:131.

92. Han H, Liu Q, Yang Z, et al. Association and cis-mQTL analysis of variants in serotonergic genes associated with nicotine dependence in Chinese Han smokers. Transl Psychiatry. 2018;8(1):243.

93. van Dongen J, Ehli EA, Jansen $\mathrm{R}$, et al. Genome-wide analysis of DNA methylation in buccal cells: a study of monozygotic twins and mQTLs. Epigenet Chromatin. 2018;11(1):54.

94. Fu X, Wang J, Du J, et al. BDNF gene's role in schizophrenia: from risk allele to methylation implications. Front Psychiatry. 2020;11:564277.

95. Purcell S, Neale B, Todd-Brown K, et al. PLINK: a tool set for wholegenome association and population-based linkage analyses. Am J Hum Genet. 2007;81(3):559-75.

96. Pan H, Holbrook JD, Karnani N, Kwoh CK. Gene, environment and methylation (GEM): a tool suite to efficiently navigate large scale epigenome wide association studies and integrate genotype and interaction between genotype and environment. BMC Bioinform. 2016;17(1):299.

97. Shabalin AA. Matrix eQTL: ultra fast eQTL analysis via large matrix operations. Bioinform Oxf Engl. 2012;28(10):1353-8.

98. Gresle MM, Jordan MA, Stankovich J, et al. Multiple sclerosis risk variants regulate gene expression in innate and adaptive immune cells. Life Sci Alliance. 2020;3(7):e202000650.

99. Millstein J, Zhang B, Zhu J, Schadt EE. Disentangling molecular relationships with a causal inference test. BMC Genet. 2009;10:23.

100. Liu Y, Aryee MJ, Padyukov L, et al. Epigenome-wide association data implicate DNA methylation as an intermediary of genetic risk in Rheumatoid Arthritis. Nat Biotechnol. 2013;31(2):142-7.

101. Min JL, Hemani G, Hannon E, et al. Genomic and phenotypic insights from an atlas of genetic effects on DNA methylation. Nat Genet. 2021;53(9):1311-21
102. Võsa U, Claringbould A, Westra H-J, et al. Large-scale cis- and trans-eQTL analyses identify thousands of genetic loci and polygenic scores that regulate blood gene expression. Nat Genet. 2021;53(9):1300-10.

103. Houseman EA, Accomando WP, Koestler DC, et al. DNA methylation arrays as surrogate measures of cell mixture distribution. BMC Bioinform. 2012;13:86.

104. Zou J, Lippert C, Heckerman D, et al. Epigenome-wide association studies without the need for cell-type composition. Nat Methods. 2014:11(3):309-11.

105. Houseman EA, Molitor J, Marsit CJ. Reference-free cell mixture adjustments in analysis of DNA methylation data. Bioinform Oxf Engl. 2014;30(10):1431-9.

106. Teschendorff AE, Breeze CE, Zheng SC, Beck S. A comparison of reference-based algorithms for correcting cell-type heterogeneity in epigenome-wide association studies. BMC Bioinform. 2017;18(1):105.

107. Teschendorff A. Epigenetic Dissection of intra-sample-heterogeneity. 2017. https://www.bioconductor.org/packages/release/bioc/html/ EpiDISH.html.

108. Zheng SC, Breeze CE, Beck S, Teschendorff AE. Identification of differentially methylated cell-types in epigenome-wide association studies. Nat Methods. 2018;15(12):1059-66.

109. Zheng S. CellDMC - a function which allows the identification of differentially methylated cell-types in Epigenome-Wide Association Studies (EWAS). 2018. https://rdrr.io/github/sjczheng/EpiDISH/man/CellDMC. html.

110. Horvath S. DNA methylation age of human tissues and cell types. Genome Biol. 2013;14(10):R115.

111. Hannum G, Guinney J, Zhao L, et al. Genome-wide methylation profiles reveal quantitative views of human aging rates. Mol Cell. 2013;49(2):359-67.

112. Zhang $\mathrm{Q}$, Vallerga $\mathrm{CL}$, Walker $\mathrm{RM}$, et al. Improved precision of epigenetic clock estimates across tissues and its implication for biological ageing. Genome Med. 2019;11(1):54.

113. Levine ME, Lu AT, Quach A, et al. An epigenetic biomarker of aging for lifespan and healthspan. Aging. 2018;10(4):573-91.

114. Yang Z, Wong A, Kuh D, et al. Correlation of an epigenetic mitotic clock with cancer risk. Genome Biol. 2016;17(1):205.

115. Lu AT, Quach A, Wilson JG, et al. DNA methylation GrimAge strongly predicts lifespan and healthspan. Aging. 2019;11(2):303-27.

116. Yang R, Wu GWY, Verhoeven JE, et al. A DNA methylation clock associated with age-related illnesses and mortality is accelerated in men with combat PTSD. Mol Psychiatry 2020;1-11.

117. Nejman D, Straussman R, Steinfeld I, et al. Molecular rules governing de novo methylation in cancer. Cancer Res. 2014;74(5):1475-83.

118. Tomasetti C, Vogelstein B. Cancer etiology. Variation in cancer risk among tissues can be explained by the number of stem cell divisions. Science. 2015;347(6217):78-81.

119. Teschendorff AE. A comparison of epigenetic mitotic-like clocks for cancer risk prediction. Genome Med. 2020;12:1-17.

120. Horvath S. DNA methylation age calculator. http://dnamage.genetics. ucla.edu/.

121. Edgar R, Domrachev M, Lash AE. Gene expression omnibus: NCBI gene expression and hybridization array data repository. Nucleic Acids Res. 2002;30(1):207-10.

122. EWAS Datahub_-National Genomics Data Center. [cited 2021 Apr 11 ]. https://bigd.big.ac.cn/ewas/datahub.

123. Xiong $Z$, Li M, Yang F, et al. EWAS Data Hub: a resource of DNA methylation array data and metadata. Nucleic Acids Res. 2020;48(D1):D890-5.

124. EWAS Atlas. [cited 2021 Apr 11]. http://bigd.big.ac.cn/ewas.

125. Li M, Zou D, Li Z, et al. EWAS Atlas: a curated knowledgebase of epigenome-wide association studies. Nucleic Acids Res. 2019;47(Database issue):D983-8.

126. Domingo-Relloso A, Huan T, Haack K, et al. DNA methylation and cancer incidence: lymphatic-hematopoietic versus solid cancers in the Strong Heart Study. Clin Epigenet. 2021;13(1):43.

127. Husquin LT, Rotival M, Fagny M, et al. Exploring the genetic basis of human population differences in DNA methylation and their causal impact on immune gene regulation. Genome Biol. 2018;19(1):222.

128. Galanter JM, Gignoux CR, Oh SS, et al. Differential methylation between ethnic sub-groups reflects the effect of genetic ancestry and environmental exposures. Elife. 2017;6:e20532. 
129. Bayega A, Fahiminiya S, Oikonomopoulos S, Ragoussis J. Current and future methods for mRNA analysis: a drive toward single molecule sequencing. Methods Mol Biol Clifton NJ. 2018;1783:209-41.

130. Nakato R, Sakata T. Methods for ChIP-seq analysis: a practical workflow and advanced applications. Methods San Diego Calif. 2021;187:44-53.

131. Zhou L, Ng HK, Drautz-Moses DI, et al. Systematic evaluation of library preparation methods and sequencing platforms for high-throughput whole genome bisulfite sequencing. Sci Rep. 2019;9(1):10383.

132. Carmona JJ, Accomando WP, Binder AM, et al. Empirical comparison of reduced representation bisulfite sequencing and Infinium BeadChip reproducibility and coverage of DNA methylation in humans. NPJ Genomic Med. 2017;2:13.

133. Simpson JT, Workman RE, Zuzarte PC, et al. Detecting DNA cytosine methylation using nanopore sequencing. Nat Methods. 2017;14(4):407-10.

\section{Publisher's Note}

Springer Nature remains neutral with regard to jurisdictional claims in published maps and institutional affiliations.

- fast, convenient online submission

- thorough peer review by experienced researchers in your field

- rapid publication on acceptance

- support for research data, including large and complex data types

- gold Open Access which fosters wider collaboration and increased citations

- maximum visibility for your research: over 100M website views per year

At BMC, research is always in progress.

Learn more biomedcentral.com/submissions 